\title{
Combinatorially-generated library of 6-fluoroquinolone analogs as potential novel antitubercular agents: a chemometric and molecular modeling assessment
}

\author{
Nikola Minovski • Andrej Perdih • Tom Solmajer
}

Received: 14 April 2011 / Accepted: 4 July 2011 /Published online: 12 August 2011

(C) Springer-Verlag 2011

\begin{abstract}
The virtual combinatorial chemistry approach as a methodology for generating chemical libraries of structurally-similar analogs in a virtual environment was employed for building a general mixed virtual combinatorial library with a total of 53.871 6-FQ structural analogs, introducing the real synthetic pathways of three well known 6-FQ inhibitors. The druggability properties of the generated combinatorial 6-FQs were assessed using an in-house developed druglikeness filter integrating the Lipinski/Veber rule-sets. The compounds recognized as drug-like were used as an external set for prediction of the biological activity values using a neural-networks (NN) model based on an experimentally-determined set of active 6-FQs. Furthermore, a subset of compounds was extracted from the pool of drug-like 6-FQs, with predicted biological activity, and subsequently used in virtual screening (VS) campaign combining pharmacophore modeling and molecular docking studies. This complex scheme, a powerful combination of chemometric and molecular modeling approaches provided novel QSAR guidelines that could aid in the further lead development of 6-FQs agents.
\end{abstract}

Keywords Antibacterial agents $\cdot$ CombiChem · DNA gyrase · Fluoroquinolones · Molecular docking · Pharmacophore modeling · QSAR · Tuberculosis

Electronic supplementary material The online version of this article (doi:10.1007/s00894-011-1179-0) contains supplementary material, which is available to authorized users.

N. Minovski $\cdot$ A. Perdih $\cdot$ T. Solmajer $(\bowtie)$

National Institute of Chemistry,

Hajdrihova 19,

1001, Ljubljana, Slovenia

e-mail: tom.solmajer@ki.si

\begin{tabular}{ll}
\multicolumn{2}{l}{ Abbreviations } \\
TB & Tuberculosis \\
ATP & Adenosine triphosphate \\
MIC & Minimal Inhibitory Concentration \\
6-FQs & 6-Fluoroquinolones \\
SAR & Structure-Activity Relationships \\
QSAR & Quantitative Structure-Activity \\
& Relationships \\
CombiChem & Combinatorial Chemistry \\
SSS & Substructure Search \\
NN & Neural-Networks \\
KANN & Kohonen Artificial Neural Networks \\
CP ANN & Counter-Propagation Artificial Neural \\
& Networks \\
GHA & Global Hypothetical Activity \\
LBP & Ligand-Based Pharmacophore \\
SBP & Structure-Based Pharmacophore \\
VS & Virtual Screening
\end{tabular}

\section{Introduction}

Tuberculosis (TB), the ingeniously transferable bacterial infection, is still one of the global health concerns [1]. Mycobaterium tuberculosis, the accountant agent of tuberculosis, is a resistful pathogen microorganism responsible for infecting about one third (two billion people) of the human population and in the process causing around two million death cases each year worldwide (World Health Organization, 2003) [2]. TB is mainly caused by the pathogen $M$. tuberculosis, but in some cases the microorganisms such as $M$. fortuitum, M. smegmatis and $M$. avium-intracellulare complex (MAC) can also be involved in the disease development [3-5]. 
The tuberculosis treatment is mainly chemotherapeutically based, with the whole therapy requiring between 6 to 9 months or even longer to be successful. The problem of drug resistance and the continuous onset of multidrug resistant lethal TB strains in most cases are attributed to the potential toxicity of the chemotherapeutics, the durability of the whole treatment, as well as frequent poor patient compliance to the therapy regimen. The increased development of resistant TB mutants is one of the additional challenging factors to stimulate the design of novel chemotherapeutic agents which will be effective against resistant Mycobacteria [6].

One of the validated and well known molecular targets of quinolone antibiotics in Mycobacteria species is the DNA gyrase which belongs to the topoisomerase group of enzymes [7]. This enzyme catalyzes the ATP dependent process of introduction of negative supercoils into closed circular DNA as well as the relaxation of the supercoiled DNA molecule (ATP-independent catalysis) [8, 9]. DNA gyrase forms a functional heterodimer $\mathrm{A}_{2} \mathrm{~B}_{2}$ consisting of two major subunits, GyrA and GyrB. The GyrA subunit is responsible for the process of breakage and reunion of the double-stranded DNA, i.e., activation of the process of DNA replication and elongation and together with GyrB is involved in the maintaining of the topological state of DNA molecule [10, 11]. Another structurally-similar enzyme which belongs to the topoisomerase group is the DNA topoisomerase IV. Like the DNA gyrase, this bacterial enzyme (a paralogue of DNA gyrase) also forms a functional heterodimer consisting of two subunits ParC and ParE (homologues of the GyrA and GyrB, respectively). Recent structural studies revealed that the quinolone antibiotics establish an interaction with the DNA breakagereunion domain of the DNA gyrase and topoisomerase IV, stabilizing the covalent topoisomerase/DNA cleavage complex, leading to a blockade of DNA replication [12].

In the last few decades, tuberculosis chemotherapy was mainly based on the active agents belonging to two main categories of antibiotics, the bacterial cell wall inhibitors (isoniazide, ethambutol) and bacterial nucleic acid synthesis inhibitors (quinolones, rifampicin) [13]. The second category of antibiotics, especially the quinolone chemical class of chemotherapeutics is increasingly gaining importance in targeting Mycobacteria, because of their effective, strong and invasive mechanism of action. Fluoroquinolones belong to the quinolone's class of DNA gyrase inhibitors which have a fluorine atom attached to the main scaffold at the 6 position (Fig. 1) [14]. The mechanism of bactericidal action is based on the inhibition of the bacterial DNA synthesis process through a scission of the natural mycobacterial DNA molecule leading to a topological stress of DNA and bacterial cell death [15].

The structure-activity relationships (SAR) studies showed that the main quinolone core (1,4-dihydro-4-oxo3-pyridinecarboxylic acid moiety) is of most significant importance for the anti-mycobacterial activity. Furthermore, unlike the cyclopropyl group at position 1 which is apparently optimal for biological activity, each substitution at positions 2, 3, and 4 will result in a significant loss of biological activity. Substitutions at positions 5 and 8 of the main quinolone core interfere with the required planarity of the system. Hydrogen and amino groups have been a good replacement for the fluorine atom at position 6 leading to an improved in vitro activity, but such modifications are not always followed by an improved in vivo activity. The substitutions at position 7 of the main scaffold are of significant importance for the biological activity as this position directly interacts with the DNA gyrase (5- or 6membered $\mathrm{N}$-hetero systems such as aminopyrrolidines and piperazines are optimal for activity) [16]. Today, the use of targeted synthetically-feasible chemical libraries significantly enhanced the hit identification as well as lead optimization phase leading to favorable novel drug candidates. Similarly, quantitative-structure activity relationship (QSAR) models proved to be useful in silico tools for the rationalization of the experimental SAR properties in the form of quantitative mathematical models which can be subsequently used for an efficient prediction of biological activity values for novel as well as unknown compounds. These tools can significantly enhance and enrich the screening process of the chemical libraries under investigation [17]. Furthermore, the three-dimensional assessment and screening of the generated molecules for optimal interaction with the binding site is of significant importance in the drug development processes [18]. For instance, ligand-based drug design approaches, such as pharmacophore modeling, which are taking into account the spatial orientation
Fig. 1 Generic structure of 6fluoroquinolone antibiotics

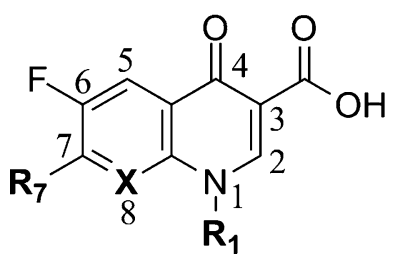

$\mathbf{R}_{\mathbf{1}}=$ usually cyclopropyl

$\mathbf{R}_{\mathbf{7}}=$ heterocyclic system

$\mathrm{X}=\mathrm{N}, \mathrm{C}-\mathrm{H}$ (or C-R) 
of the ligand's functional groups and scaffold shape complementarity can be utilized for the construction of predictive pharmacophore models which can be more effective for virtual screening (VS). Such a model based on the similarity of the pharmacophoric features is particularly useful, if it is able to identify (recognize) active compounds among a pool of inactive molecules. Moreover, the availability of three-dimensional (3D) structure of the protein-ligand complex of interest enables the implementation of the structure-based approaches especially molecular-docking calculations of ligands into the defined binding site of the protein and the investigation of ligand-protein molecular interactions $[19,20]$.
The present study introduces an effective methodology for the in silico generation of novel unknown 6-fluoroquinolone (6-FQ) analogs using a combinatorial chemistry approach coupled with the prediction of values of biological activity by employing a previously derived neural-networks (NN) [21]. Furthermore, a three-dimensional pharmacophore approach and molecular docking calculations were used to assess the generated virtual library utilizing the available experimental data to select the most promising drug-like compounds. This scheme - a powerful combination of the chemometric and VS tools - is aimed to establish new possible SAR guidelines and trends in 6-fluoroquinolone optimization, which could be of importance in the on-going antibacterial lead discovery programs (Fig. 2).

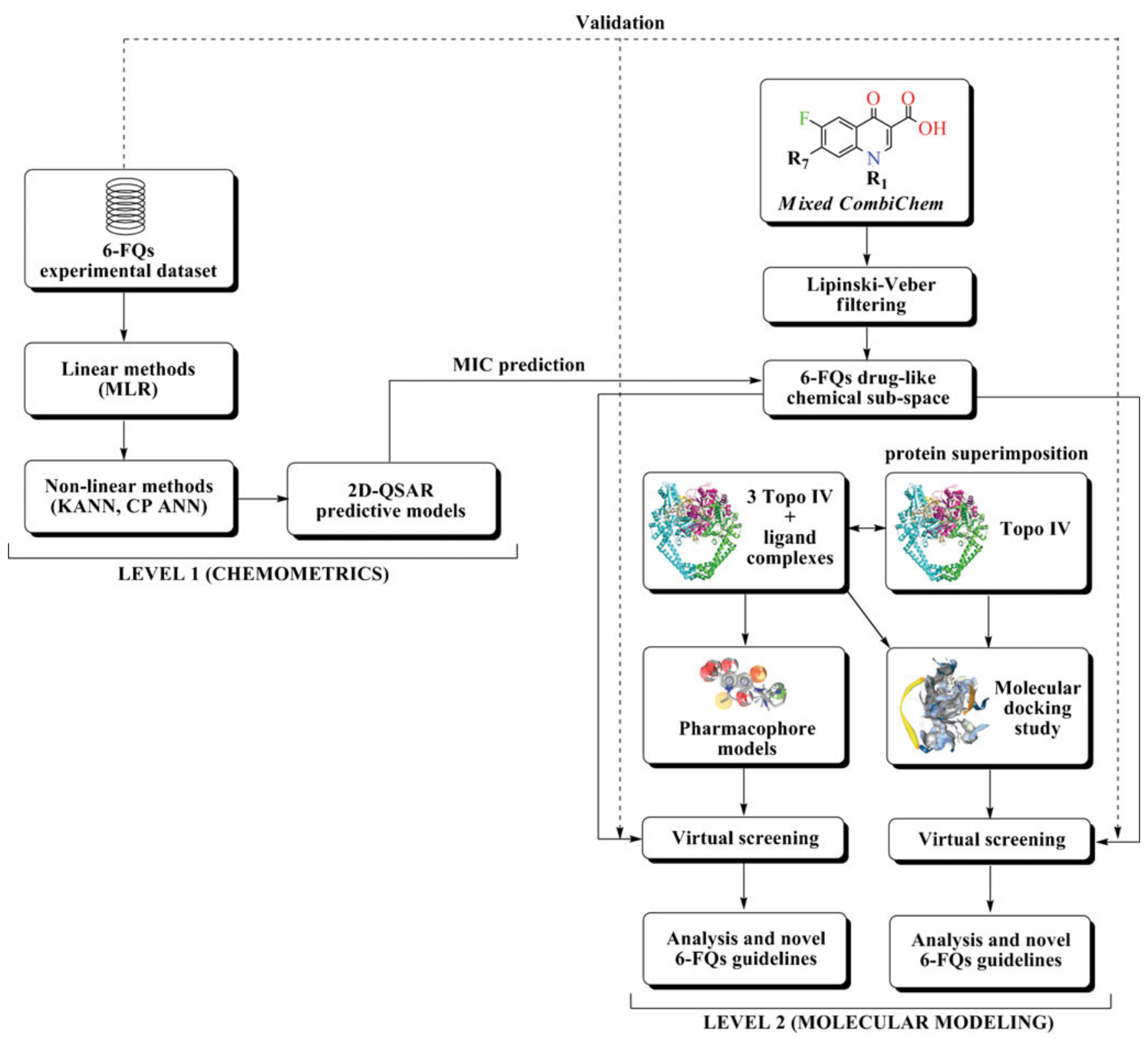

Fig. 2 The overall workflow depicting different stages of the performed chemometric and molecular modeling study 


\section{Computational details}

Virtual combinatorial generation

One of the most popular strategies in modern drug design is the virtual synthesis of analogs of existing compounds for which the bioavailability and toxicity studies are already performed and have displayed activity and potency in human therapy. The implementation of SAR-based scaffold modifications to obtain novel molecules for the target under investigation is a well-known and advantageous strategy for analog design. There are several software tools available integrating the combinatorial algorithm for in silico virtual combinatorial generation. CombiChem add-on modul available in ChemBioOffice Ultra was used for in silico generation of 6-fluoroquinolone structural analogs [22]. For this purpose we employed the original synthetic pathways of three well known 6-FQ antibiotics: ciprofloxacin, moxifloxacin, and ofloxacin [23-25]. Using a building-blocks commercial dataset (Bionet Fragment Library of 6995 "Rule of 3" filtered lead-like fragments) [26], and SAR-based structural modifications at positions 1 and 7 of the main 6-FQ scaffold, we built six different subsets of combinatorially-generated 6-fluoroquinolone analogs.

The virtual environment enables a rational simplification of the real synthetic reactions taking into account only the crucial synthetic steps for derivation of the final product (structurally-similar 6-FQ analogs). Such a rational virtual simplification resulted in two-steps virtual synthetic pathways for ciprofloxacin and moxifloxacin (yielding $\mathrm{R}_{1}$-monosubstituted intermediate product, as well as all possible $\mathrm{R}_{1}, \mathrm{R}_{7}$-disubstituted 6-FQ analogs as final products), and one-step virtual synthetic pathway for ofloxacin (obtaining all possible $\mathrm{R}_{7}$-monosubstituted analogs as final products). Taking into account that position 7 of the main 6-FQ moiety directly interacts with the DNA gyrase and is of significant importance for activity [16], additionally we introduced several different substituents at this position of the main scaffold with nonamine attachment points. These substructural modifications enable a total of six virtual combinatorial synthetic mechanisms to be defined (Fig. 3):

(1) 7-amino substituted ciprofloxacin's structural analogs.

(2) 7-non-amino substituted ciprofloxacin's structural analogs.

(3) 7-amino substituted moxifloxacin's structural analogs.

(4) 7-non-amino substituted moxifloxacin's structural analogs.

(5) 7-amino substituted ofloxacin's structural analogs.

(6) 7-non-amino substituted ofloxacin's structural analogs.
Fragments selection for combinatorial enumeration

A building-blocks commercial library of 6995 substructural fragments was used as a source for the fragment selection for combinatorial enumeration [26]. Each fragment in such a library is defined by the "rule of 3 " (MW $\leq 300$; $n \mathrm{HBD}$, $n \mathrm{HBA}, n \mathrm{RB} \leq 3$ ), where MW is the molecular weight, while $n \mathrm{HBD}, n \mathrm{HBA}$, and $n \mathrm{RB}$, are number of hydrogen bond donors, number of hydrogen bond acceptors, and number of rotatable bonds, respectively [27]. Fragments selection was performed by employing the substructure search (SSS) algorithm. The selection procedure resulted in the extraction of all possible substructural fragments (primary amines and secondary amines/non-amines) defined by the virtual synthetic pathways. Substructural fragmental subsets selected by the SSS algorithm for each virtual synthetic pathway were visually inspected for the presence of possible unwanted species (salt forms, mixtures, charged forms). These substructural fragments were excluded from the combinatorial procedure implementing a simple Boolean filtering algorithm (Y/N (Yes/No)). Furthermore, all substructural fragments containing more than one attachment point (two or more amino groups) were eliminated. The rest of the substructural fragments with $(\mathrm{Y})$ output obtained using this filtering procedure (described in details in Table 1 for each virtual combinatorial definition), were used as a starting point for combinatorial enumeration.

\section{Combinatorial enumeration}

The combinatorial enumeration procedure was performed by implementing a standard methodology [28] for generating structural analogs in virtual environment (CombiChem) [22]. This procedure of statistical non-repetitive fragmental permutation of the selected building-blocks subsets $\left(\mathrm{R}_{1}\right.$ : primary amines and $\mathrm{R}_{7}$ : secondary amines/non-amines)) in previously defined variable positions ( 1 and 7) within the main 6-FQ scaffold generated all possible virtual 1,7 substituted 6-FQ structural analogs. The total number of analogs $\left(\rho_{t o t}\right)$ obtained using this methodology (Table 1), mathematically can be estimated as a multiplication product between the total number of selected substructural fragments permutating at position $1\left(\mathrm{R}_{1}\right.$ : primary amines, $\left.N_{i}\right)$ and total number of selected substructural fragments permutating at position 7 $\left(\mathrm{R}_{7}\right.$ : secondary amines/non-amines, $\left.M_{j}\right)$ within the main 6-FQ core, using the following equation (Eq. 1) [29]:

$\rho_{\text {tot }}=N_{i} \times M_{j}$

The obtained products $\left(\rho_{t o t}=53.871\right)$ belong to three main categories of 6-FQ analogs: ciprofloxacin analogs (7-amino substituted analogs (12.296), 7-non-amino substituted analogs (21.965)), moxifloxacin analogs (7-amino substituted 
A) Virtual synthetic pathway for combinatorial enumeration of ciprofloxacin analogs:<smiles>CN=CC(C(=O)OC)C(=O)c1cc(F)c(F)cc1F</smiles>

starting material

$$
\mathbf{R}_{1}
$$
$\mathrm{NH}_{2}$

$\left[N_{i}\right]$<smiles>[R1]n1cc(C(=O)OC)c(=O)c2cc(F)c(F)cc21</smiles>

$R_{1}$-monosubstituted intermediate

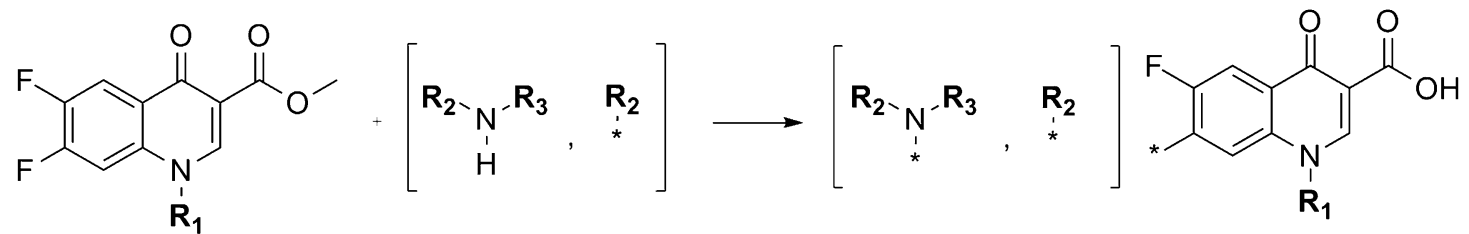

$\left[M_{j}\right]$ $\left[\mathrm{CIP}-N_{i}-M_{j}, \mathrm{CIP}^{\prime}-N_{i}-M_{j}\right]$

B) Virtual synthetic pathway for combinatorial enumeration of moxifloxacin analogs:<smiles>CCOC=C(C(=O)OCC)C(=O)c1cc(F)c(F)c(OC)c1F</smiles>

starting material<smiles>[R]N=N</smiles>

$\left[N_{i}\right]$

\section{$\mathbf{R}_{\mathbf{1}}$-monosubstituted intermediate}<smiles>[R]n1cc(C(=O)O)c(=O)c2cc(F)c(F)c(OC)c21</smiles><smiles>[R]NN[R]C1CC1CC</smiles>

$\left[M_{j}\right]$

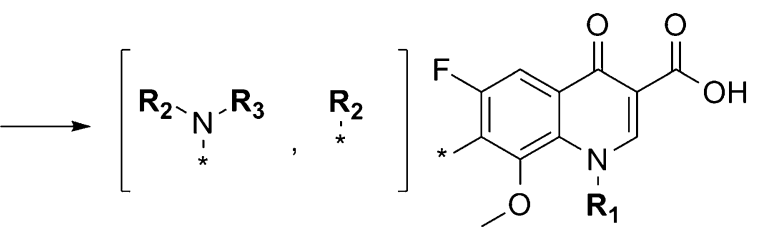

$\mathbf{R}_{\mathbf{1}}, \mathbf{R}_{\mathbf{7}}$-disubstituted moxifloxacin analogs $\left[\operatorname{MOX}-N_{i}-M_{j}, \mathrm{MOX}^{\prime}-N_{i}-M_{j}\right]$

C) Virtual synthetic pathway for combinatorial enumeration of ofloxacin analogs:<smiles>CC1COc2c(F)c(F)cc3c(=O)c(C(=O)O)cn1c23</smiles>

starting material<smiles>[R]C1CCCC1[R]</smiles>

$\left[\boldsymbol{M}_{j}\right]$

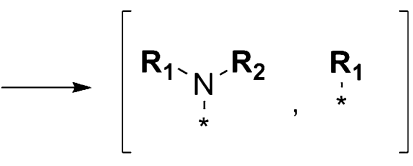<smiles>Cc1c(F)cc2c(=O)c(C(=O)O)cn3c2c1OCC3C</smiles>

$\mathbf{R}_{\mathbf{7}}$-monosubstituted ofloxacin analogs [OFL- $M_{j}$, OFL'- $M_{j}$ ]

Fig. 3 Generic virtual synthetic pathways for combinatorial enumeration of three different 6-FQs: (A) ciprofloxacin analogs $\left(\mathrm{CIP}-N_{i}-M_{j}\right.$, CIP'- $N_{i}{ }^{-}$ $\left.M_{j}\right)$, (B) moxifloxacin analogs $\left(\mathrm{MOX}-N_{i}-M_{j}, \mathrm{MOX}^{\prime}-N_{i}-M_{j}\right)$, (C) ofloxacin analogs $\left(\mathrm{OFL}-M_{j}\right.$, OFL'- $\left.M_{j}\right)$

analogs (8.510), 7-non-amino substituted analogs (10.731)), and ofloxacin analogs (7-amino substituted analogs (180), 7-non-amino substituted analogs (189)). The following designation scheme was used: ciprofloxacin analogs (7-amino substituted analogs $\left(\mathrm{CIP}-N_{i}-M_{j}\right)$, 7-non-amino substituted analogs $\left(\mathrm{CIP}^{\prime}-N_{i}-M_{j}\right)$ ), moxifloxacin analogs (7amino substituted analogs (MOX- $\left.N_{i}-M_{j}\right)$, 7-non-amino substituted analogs $\left(\mathrm{MOX}^{\prime}-N_{i}-M_{j}\right)$ ), and ofloxacin analogs (7amino substituted analogs (OFL- $\left.M_{j}\right)$, 7-non-amino substituted analogs $\left.\left(\mathrm{OFL}^{\prime}-M_{j}\right)\right)$. Combining all subsets of analogs 
Table 1 Fragments selection details for combinatorial enumeration. The building-blocks $\left(\mathrm{R}_{7}\right.$-substructural fragments) with non-amino attachment point are signed with asterisk $(*)$

\begin{tabular}{|c|c|c|c|c|c|c|c|c|c|}
\hline \multirow[t]{3}{*}{ ID } & \multicolumn{2}{|c|}{ Virtual combinatorial definition } & \multicolumn{2}{|c|}{ Substructural fragments } & \multicolumn{4}{|c|}{ Boolean output $(\mathrm{Y} / \mathrm{N})$} & \multirow[t]{3}{*}{$\rho_{t o t}$} \\
\hline & \multirow[t]{2}{*}{$\mathrm{R}_{1}$} & \multirow[t]{2}{*}{$\mathrm{R}_{7}$} & \multirow[t]{2}{*}{$\mathrm{R}_{1}$} & \multirow[t]{2}{*}{$\mathrm{R}_{7}$} & \multicolumn{2}{|l|}{$\mathrm{R}_{1}$} & \multicolumn{2}{|l|}{$\mathrm{R}_{7}$} & \\
\hline & & & & & $\mathrm{Y}\left(N_{i}\right)$ & $\mathrm{N}$ & $\mathrm{Y}\left(M_{j}\right)$ & $\mathrm{N}$ & \\
\hline 1. & $\mathrm{R}_{1}-\mathrm{NH}_{2}$ & $\mathrm{R}_{2} \mathrm{R}_{3}-\mathrm{NH}$ & 126 & 363 & 116 & 10 & 106 & 257 & 12.296 \\
\hline 2. & $\mathrm{R}_{1}-\mathrm{NH}_{2}$ & $\mathrm{R}_{2^{-*}}$ & 123 & 340 & 115 & 8 & 191 & 149 & 21.965 \\
\hline 3. & $\mathrm{R}_{1}-\mathrm{NH}_{2}$ & $\mathrm{R}_{2} \mathrm{R}_{3}-\mathrm{NH}$ & 123 & 347 & 115 & 8 & 74 & 273 & 8.510 \\
\hline 4. & $\mathrm{R}_{1}-\mathrm{NH}_{2}$ & $\mathrm{R}_{2^{-}} *$ & 123 & 337 & 73 & 50 & 147 & 190 & 10.731 \\
\hline 5. & N/A & $\mathrm{R}_{1} \mathrm{R}_{2}-\mathrm{NH}$ & $\mathrm{N} / \mathrm{A}$ & 348 & N/A & $\mathrm{N} / \mathrm{A}$ & 180 & 168 & 180 \\
\hline 6. & N/A & $\mathrm{R}_{1^{-}} *$ & N/A & 337 & N/A & N/A & 189 & 148 & 189 \\
\hline
\end{tabular}

obtained by using the combinatorial algorithm, resulted in building a general virtual combinatorial library (CombiTot) with a total of 53.8716 -FQ structural analogs, which were subsequently used for assessing the drug-like properties (all structures are available as *.sdf file format in electronic supplementary material, online resource 1).

\section{Drug-likeness assessment using chemometric approaches}

Albeit this combinatorial library contains a considerable number of 6-FQ analogs (CombiTot, $\rho_{\text {tot }}=53.871$ ) it can be considered as a small sub-space in the available chemical space [30]. Since the combinatorial algorithm in first instance increased the molecular complexity, there is a low probability that each compound in such a virtual library will possess drug-like properties. Therefore, drug-likeness filtering was performed taking into consideration the rules of Lipinski [31] and Veber [32]. Using the ChemBioOffice pre-integrated cheminformatics functions [22], we developed a robust drug-likeness filtering tool integrating both rule-sets (Lipinski "rule of 5" and Veber rules) which was used for filtering of our general combinatorial library and defining the drug-like chemical sub-space of 6-FQ analogs. This filtering procedure yielded a list of 1.101 out of 53.871 virtual combinatorial 6-FQ analogs as promising compounds for further investigation. These compounds were further assessed for prediction of their unknown activity values $\left(p \mathrm{MIC}_{\text {pred-combi }}\right)$ using a prebuilt QSAR model [21]. Combinatorially-generated 6-FQ analogs possessing drug-like properties (CombiDL, 1.101 analogs) are available as "electronic supplementary material" (*.sdf file format, online resource 2).

Prediction of the biological activity values using a derived non-linear neural networks (NN) model

Our previously published non-linear neural networks (NN) model (see [21] for details) was used for prediction of biological activity values $\left(p \mathrm{MIC}_{\text {pred-combi }}\right)$ for the external combinatorial library of 1.101 novel unknown 6-FQ structural analogs. The model was built employing counter-propagation artificial neural networks (CP ANN) methodology using a dataset of 145 structurally-similar 6-fluoroquinolones (all analogs are available as *.sdf file format in electronic supplementary material, online resource 3) with experimentally-determined biological activity values $\left(p \mathrm{MIC}_{\text {exp }}\right)$ against $M$. tuberculosis and an extensive set of nearly 600 calculated 2D molecular descriptors. Heuristic algorithm and intercorrelation matrix were used for selection of statistically-significant molecular descriptors for activity. The Heuristic algorithm which is a suitable method for preselection of molecular descriptors is first calculating the oneparameter correlation equations between molecular descriptors and activity, eliminating all descriptors that do not fulfill the pre-defined criteria: (1) The Fisher $F$-test value for the one-parameter correlation with the descriptor is less than $1.00,(2)$ The squared correlation coefficient $\left(R^{2}\right)$ for the oneparameter equation is less than $R_{\min }{ }^{2}$ (the default value is $R_{\text {min }}{ }^{2}=0.1$ ), (3) $t$-test value is less than $t 1$ (where $R_{\min }{ }^{2}$ and $t 1=1.5$ ), and (4) the descriptor is highly intercorrelated with another descriptor (above $r_{\text {full }}$, where $r_{\text {full }}=0.99$ ), and this other descriptor has a higher squared correlation coefficient in the one-parameter equations based on these descriptors. With the remaining descriptors, the algorithm calculates all possible two- and more-parameter linear models [33]. Initially, several linear models with up to ten descriptors were developed. The frequency analysis of occurrence of the molecular descriptors between these models, resulted in selection of the most frequently occuring molecular descriptors which were used as input variables in neural-networks modeling part. Kohonen artificial neural networks (KANN) was employed for splitting the dataset into a training set (Assay2, 115 compounds) and an external validation set (Assay2, 30 compounds). The model was built on the training set using 
CP ANN and internally validated employing the crossvalidation leave-one-out procedure $\left(R_{t r}=0.96, R_{t r-c v}=0.62\right.$, where $R_{t r}$ designates the coefficient of correlation for the model, while $R_{t r-c v}$ is coefficient of correlation for crossvalidation leave-one-out) as well as externally validated for its predictive performances using the external validation set $\left(R_{v a l}=0.8454\right.$, where $R_{\text {val }}$ is coefficient of correlation for the external validation set). Selection of a subset of combinatorially-generated 6-FQ analogs for pharmacophore analysis was performed by defining a global hypothetical activity (GHA) range $0.00 \leq \mathrm{MIC}_{\text {pred- }}$ combi $[\mu \mathrm{g} / \mathrm{mL}] \leq 0.10$ based on the determined experimental data for the following 6-FQs: Structure2 (levofloxacin, $\mathrm{MIC}_{\text {exp }}=0.0115 \mu \mathrm{g} / \mathrm{mL}$ ), Structure 1 (clinafloxacin, $\mathrm{MIC}_{\text {exp }}=0.01 \mu \mathrm{g} / \mathrm{mL}$ ), and Structure121 (moxifloxacin, $\mathrm{MIC}_{\text {exp }}=0.025 \mu \mathrm{g} / \mathrm{mL}$ ) [21]. This selection resulted in the extraction of 427 out of $1.1016-\mathrm{FQ}$ analogs which had their activity predicted inside the pre-defined GHA range. These analogs were used as a drug-like combinatorial library (CombiLib) of novel 6-FQs for three-dimensional pharmacophore analysis and molecular docking study (all structures are available as *.sdf file format in electronic supplementary material, online resource 4).

\section{Ligand-based and structure-based pharmacophore modeling of the 6-fluoroquinolone analogs}

Although both DNA gyrase A (GyrA) and B (GyrB) subunits have been solved by the $\mathrm{x}$-ray crystallography [34, 35], currently a full experimental atomistic picture of the $\mathrm{GyrA}_{2} \mathrm{GyrB}_{2}$ tetramer in complex with the DNA and 6-fluoroquinolone molecules remains unidentified. On the other hand, recent seminal studies on the closely related type II topoisomerase - topoisomerase IV - the complexes formed by the Streptococcus pneumoniae ParC (equivalent of GyrA subunit) and ParE TOPRIM domains of the topoisomerase IV together with 6-fluoroquinolones intercalated in the gap between nucleotides of the DNA were solved [36]. These data provided the first solid atomistic insights into the mechanism of action of the fluoroquinolone antibacterial agents. As both type II topoisomerases (DNA gyrase and topoisomerase IV) share a close structural and functional resemblance we used both available crystal structures to perform pharmacophore modeling and molecular docking calculations. Furthermore, for the 6-FQ chemical class a good correlation between the measured in vitro $\mathrm{IC}_{50}$ values and in vivo MIC activities was observed. This enables utilization of the in vivo MIC data also for the interpretation of the atomistic pharmacophore and docking calculations [37].

Pharmacophore modeling approach is one of the widely used concepts in modern drug discovery [34]. Several software tools available today are able to provide predictive
3D pharmacophore models in an automated fashion. LigandScout [18], a software tool for automatic pharmacophore model generation from the available structural information was employed for the visualization and exploration of the topoisomerase IV binding site in complex with known 6-FQ active agents (structure-based design approach) as well as for constructing 3Dpharmacophore models based only on the 6-FQ ligand structures (ligand-based design approach). Three ligandtopoIV-DNA cleavage complexes from $S$. pneumoniae, available from the Protein Data Bank (PDB) repository [pdb codes: 3FOE, 3FOF, 3K9F], were used for the 3Dpharmacophore models generation [36, 38]. The models were obtained by automatic recognition of the 6-FQ cocrystallized ligand structures (clinafloxacin (3FOE), moxifloxacin (3FOF), and levofloxacin (3K9F)) and the surrounding amino acid residues of the 6-FQ analogs binding site and by analysis of possible ligand-protein interactions. Subsequently, the 3D structure-based pharmacophore (SBP) models were generated automatically (assignment of the pharmacophoric features for each ligand separately and ligand alignment generation) together with excluded volume spheres.

Therefore, two structure-based pharmacophore (SBP) models: shared $\left(\mathrm{SBP}_{\text {shared }}\right.$, interpolation of the identified pharmacophore features) and merged ( $\mathrm{SBP}_{\text {merged }}$, unification of the identified pharmacophore features) were constructed. Furthermore, the experimental conformations of the three bound 6-FQ analogs, were used for building of ligand-based pharmacophore (LBP) model. For each of the molecules, 250 unique conformations were calculated and aligned to yield a ligand-based pharmacophore (LBP) model. Finally, both combinatorial (CombiLib) and experimental library (Assay 2) were converted into multiconformer libraries (maximum 25 conformations for each molecule) and subsequently screened with built-in LigandScout pharmacophore VS engine to evaluate the matching of the investigated compounds to the derived set of pharmacophore features.

Molecular docking of the 6-fluoroquiniolone analogs

\section{Molecular docking of the 6-fluoroquinolone molecules into the type II topoisomerase crystal structure}

The docking experiments were performed by using GOLD docking engine [39], and protein structure with the PDB code $3 \mathrm{~K} 9 \mathrm{~F}$ was used to define the binding site located around the experimental coordinates of the bound 6-FQ inhibitor levofloxacin resulting in a cavity radius of $12.5 \AA$. Each investigated molecule was docked 10 times into the binding site by applying the following parameters of the GOLD genetic algorithm (GA) (population size $=100$, 
selection pressure $=1.1$, no. of operations $=100000$, no. of islands $=5$, niche size $=2$, migrate $=10$, mutate $=95$, crossover $=95$ ). Early termination was allowed if the top three solutions were within $1.5 \AA$ of the root-mean-squareddeviation (RMSD) value. For the assessment of the binding affinity, GOLDscore scoring function was used [39].

The quality of the generated binding poses were validated by re-docking the levofloxacin into its binding site and exploring the positioning of the 1456 -FQ molecules with the available experimental MIC data [40]. Finally, all compounds of the generated reduced combinatorial library (CombiLib, 427 compounds, online resource 4), were docked into the defined binding site using the same procedure.

\section{Results and discussion}

Virtual combinatorial chemistry approach as a strategy for generating chemical libraries of structurally-similar analogs for a given compound of interest as well as their investigation implementing highly-sophisticated cheminformatics algorithms are extensively documented [41-45]. There are two crucial steps which must be taken into account for combinatorial generation of structural analogs for the entity under investigation: the synthetic procedure (generic synthetic reaction) for preparation of the compound as well as the introduction of substituents onto a scaffold [26]. In our case, a focused virtual combinatorial library of novel 6-FQ analogs (CombiTot) utilizing the established combinatorial algorithm for SAR-based scaffold permutation was obtained.

Druggability properties assessment and defining the drug-like chemical sub-space

The drug-likeness assessment of the combinatoriallygenerated library of 6-FQ structural analogs (CombiTot, 53.871 compounds) was carried out by calculating the druggability properties: MW, AlogP (Ghose-Crippen's atom-based model for $\log \mathrm{P}$, i.e., partition coefficient for $n$-octanol/water bi-phase system), $n \mathrm{HBD}, n \mathrm{HBA}, \mathrm{MPSA}$ (molecular polar surface area), and $n \mathrm{RB}$.

The calculated druggability properties were analyzed using the property distribution comparison methodology (histogram-type of analysis, Table 2, Fig. 4) [46] between the dataset of known 6-FQs used in the development of the NN model (Assay2, 145 compounds, Fig. 4A) [21] and the combinatorial (CombiTot, 53.871 compounds, Fig. 4B).

The results of the statistical analysis show that only one of the calculated properties for the experimental compounds (Fig. $4 \mathrm{~A})$ follows normal Gaussian distribution $\left(n \mathrm{HBD}_{\text {exp }}\right)$, whereas the rest of the calculated properties $\left(\mathrm{MW}_{\text {exp }}\right.$, $\mathrm{MPSA}_{\text {exp }}, \mathrm{Alog}_{\text {exp }}, n \mathrm{HBA}_{\text {exp }}$, and $\left.n \mathrm{RB}_{\text {exp }}\right)$ are asymmetrically distributed. The peak-analysis (the top-point on the
Gaussian curve where the distribution of the calculated property reaches $50 \%$ ) showed acceptable values for druglikeness: $\mathrm{MW}_{\text {exp }}=468, \mathrm{~A} \log \mathrm{P}_{\text {exp }}=-0.07, n \mathrm{HBD}_{\text {exp }}=2$, $n \mathrm{HBA}_{\text {exp }}=8, \quad \mathrm{MPSA}_{\text {exp }}=103$, and $n \mathrm{RB}_{\text {exp }}=5$. The corresponding mid-50\% values (the interval between $25 \%$ and $75 \%$ of the distribution, i.e., first (Q1) and third (Q3) calculated quartile, respectively) were obtained employing the quartile calculation: $\mathrm{MW}_{\text {exp-mid50\% }}=382-566, A \log \mathrm{P}_{\text {exp- }}$ mid50\% $=(-0.848)-(+0.394), n \mathrm{HBD}_{\text {exp-mid } 50 \%}=2, n \mathrm{HBA}_{\text {exp- }}$ mid50\% $=6-9, \mathrm{MPSA}_{\text {exp-mid } 50 \%}=74.38-124.77$, and $n \mathrm{RB}_{\text {exp- }}$ mid $50 \%=3-6$ (Table 2A). These results are in accordance with the Lipinski and Veber rule-sets, respectively, and clearly define the domain in which the compounds possess drug-like characteristics [31, 32].

On the other hand, an obvious difference in the property distribution was observed for the compounds within the virtual combinatorial set (Fig. 4B). A normal Gaussian distribution was observed for five calculated properties $\left(\mathrm{MW}_{\text {combi }}\right.$,

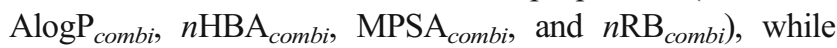
the $n \mathrm{HBD}_{\text {combi }}$ parameter follows an asymmetric distribution. The peak-analysis for the combinatorial set resulted in following values: $\mathrm{MW}_{\text {combi }}=621, \mathrm{~A} \log \mathrm{P}_{\text {combi }}=4.87$, $n \mathrm{HBD}_{c o m b i}=2, n \mathrm{HBA}_{c o m b i}=9, \quad \operatorname{MPSA}_{c o m b i}=157$, and $n \mathrm{RB}_{\text {combi }}=7$, whereas the corresponding mid- $50 \%$ values were in the following boundaries: $\mathrm{MW}_{\text {combi-mid } 50 \%}=578$

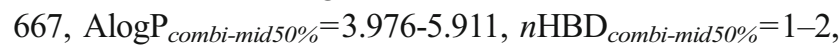
$n \mathrm{HBA}_{\text {combi-mid } 50 \%}=8-10$, MPSA $_{\text {combi-mid } 50 \%}=137.36-174.89$, and $n \mathrm{RB}_{\text {combi-mid } 50 \%}=6-8$ (Table 2B).

The increased values $\left(\mathrm{MW}_{\text {combi }}\right.$ and MPSA $\left.\mathrm{A}_{c o m b i}\right)$ pointed toward the increased molecular complexity [47] and a decreased probability for a good ligand-protein interaction [48]. Thus, a pre-filtering of the combinatorial library was performed by implementing a comprehensive Boolean-type ( $\mathrm{T} / \mathrm{F}$ (true/false)) drug-likeness filtering algorithm (CombiVL; $\mathrm{MW}<500, \mathrm{~A} \log \mathrm{P}<5.0, n \mathrm{HBD} \leq 5, n \mathrm{HBA} \leq 10$, MPSA $\leq 140, n \mathrm{RB} \leq 10$ ) integrating both rule-sets (Lipinski "rule-of-five" and Veber rules) [31, 32].

Such a filtering procedure resulted in eliminating all 6-FQ analogs which do not satisfy the above criteria (in the CombiTot library 52.770 eliminated compounds were marked as false (F)). The retained 6-FQ structural analogs (total 1.101 compounds) define the drug-like chemical subspace (CombiDL, online resource 2) which was subsequently used for prediction of the biological activity values $\left(p \mathrm{MIC}_{\text {pred-combi }}\right)$ employing our derived NN model [21].

Prediction of the biological activity values for the novel combinatorially-generated 6-fluoroquinolone drug-like analogs and activity-based subset selection

A derived seven parameter neural-networks (NN) model (Assay2, 145 compounds, online resource 3) [21] was used for prediction of the biological activity values $\left(p \mathrm{MIC}_{\text {pred-combi }}\right)$ 
Table 2 Property distribution analysis of the experimental and the combinatorial set, respectively (No., number of compounds in the dataset; Prop., calculated property; Mean, the mean value; Min, minimum value; Q1, first quartile; Median, the median value; Q3,

\begin{tabular}{|c|c|c|c|c|c|c|c|c|}
\hline A) & No. & Prop. & Mean & Min & Q1 & Median & Q3 & Max \\
\hline \multirow[t]{6}{*}{ Experimental set } & \multirow[t]{6}{*}{145} & $\mathrm{MW}_{\exp }$ & 467.968 & 275.280 & 382.380 & 419.420 & 566.490 & 801.240 \\
\hline & & $\mathrm{A} \log \mathrm{P}_{\exp }$ & -0.069 & -2.453 & -0.848 & -0.337 & 0.394 & 6.251 \\
\hline & & $n \mathrm{HBD}_{\exp }$ & 1.952 & 1 & 2 & 2 & 2 & 3 \\
\hline & & $n \mathrm{HBA}_{\exp }$ & 7.621 & 4 & 6 & 7 & 9 & 14 \\
\hline & & MPSA $_{\exp }$ & 102.983 & 57.610 & 74.380 & 86.150 & 124.770 & 186.310 \\
\hline & & $n \mathrm{RB}_{\exp }$ & 4.959 & 1 & 3 & 5 & 6 & 11 \\
\hline B) & No. & Prop. & Mean & Min & Q1 & Median & Q3 & Max \\
\hline \multirow[t]{6}{*}{ Combinatorial set } & \multirow[t]{6}{*}{53.871} & $\mathrm{MW}_{c o m b i}$ & 621.099 & 329.280 & 578.410 & 623.540 & 666.620 & 814.440 \\
\hline & & $\mathrm{A} \log \mathrm{P}_{c o m b i}$ & 4.872 & -3.735 & 3.976 & 5.002 & 5.911 & 9.265 \\
\hline & & $n \mathrm{HBD}_{c o m b i}$ & 1.958 & 1 & 1 & 2 & 2 & 6 \\
\hline & & $n \mathrm{HBA}_{c o m b i}$ & 8.868 & 4 & 8 & 9 & 10 & 13 \\
\hline & & MPSA $_{c o m b i}$ & 156.742 & 65.780 & 137.360 & 155.030 & 174.890 & 272.820 \\
\hline & & $n \mathrm{RB}_{\text {combi }}$ & 6.807 & 2 & 6 & 7 & 8 & 12 \\
\hline
\end{tabular}

for the previously filtered combinatorially-generated druglike 6-FQ structural analogs (CombiDL, 1.101 compounds, online resource 2). Using this developed model the biological activity $\left(p \mathrm{MIC}_{\text {pred-combi }}\right)$ in the series of novel unknown 6-FQ analogs is correlated to a set of seven constitutional, topological, and electrostatic parameters (Table 3).

As presented in Table 3, nR09 belongs to the class of pure constitutional parameters, GATS8v, YZS/YZR, X1A, and $\mathrm{PW}$, are molecular descriptors which belong to the class of topological parameters, while the parameters JGI2 and JGI3, belong to the class of pure electrostatic parameters. These molecular descriptors, which in general accentuate the importance of molecular shape can be linked to the accommodation of the main 6-FQ scaffold within the GyrA subunit binding pocket. Since the QSAR model was built employing a series of structurally-similar 6-FQ analogs with experimentally determined biological activity values, one would expect similar biological response of these compounds within the same protein target. In this respect, we believe that GATS8v parameter alone, as molecular descriptor describing the importance of the atomic van der Waals volumes of our 6-FQs, is of particular significance for the biological activity, mainly through establishing proper steric complementarity between the ligand and the enzyme. On the other hand, the X1A parameter is a typical topological molecular descriptor, i.e., a pharmacophore fingerprint that carries the connectivity information of 6-FQ analogs, probably through establishing $\pi-\pi$ stacking interactions between the planar aromatic/heteroaromatic systems in the 6-FQs and the GyrA/DNA, respectively.

The electrostatic descriptors JGI2 and JGI3 suggest that anti-mycobacterial activity is potentially dependent on the charge indices for the oxygen of the carboxyl and carbonyl third quartile; Max, the maximum value). (A) the experimental set (145 compounds), (B) the combinatorial set (CombiTot, 53.871 compounds) possibility for establishing hydrogen-bonding interactions between these substituents and the amino acid residues within the GyrA binding pocket. Moreover, these parameters suggest the possibility of establishing an electrostatic interaction between the $\mathrm{F}$ atom at position 6 and the target protein which may result in an enhanced binding of the 6-FQ analogs to the complex.

\section{Activity-based subset selection for molecular modeling calculations}

The predicted biological activity values $\left(p \mathrm{MIC}_{\text {pred-combi }}\right)$ for the combinatorially-generated drug-like 6-FQ analogs are in the range between $0.0125<p \mathrm{MIC}_{\text {pred-combi }}<0.9174$, while the corresponding $\mathrm{MIC}_{\text {pred-combi }}$ values, obtained after $p \mathrm{MIC}_{\text {pred- }}$ combi de-normalization and anti-logarithmization, are in the range between $0.0021<\mathrm{MIC}_{\text {pred-combi }}[\mu \mathrm{g} / \mathrm{mL}]<6.3726$. Using a GHA range described previously, a subset of $4276-\mathrm{FQ}$ analogs (CombiLib, online resource 4) was extracted from the pool of total 1.101 compounds (CombiDL). Such a selection procedure ensures that each 6-FQ analog in the MIC-based isolated subset (CombiLib) possess biological activity $\left(0.4809<p \mathrm{MIC}_{\text {pred-combi }}<0.9174 ; 0.0021<\mathrm{MIC}_{\text {pred-combi }}\right.$ $[\mu \mathrm{g} / \mathrm{mL}]<0.1000)$ against $M$. tuberculosis. The selected combinatorial subset (CombiLib, 427 compounds), was subsequently used as an external library in the three-dimensional pharmacophore analysis and molecular docking study.

\section{6-FQs pharmacophore modeling study}

Using LigandScout's the integrated automatic pharmacophore generation algorithm, three pharmacophore models group within the main core. They also describe the 

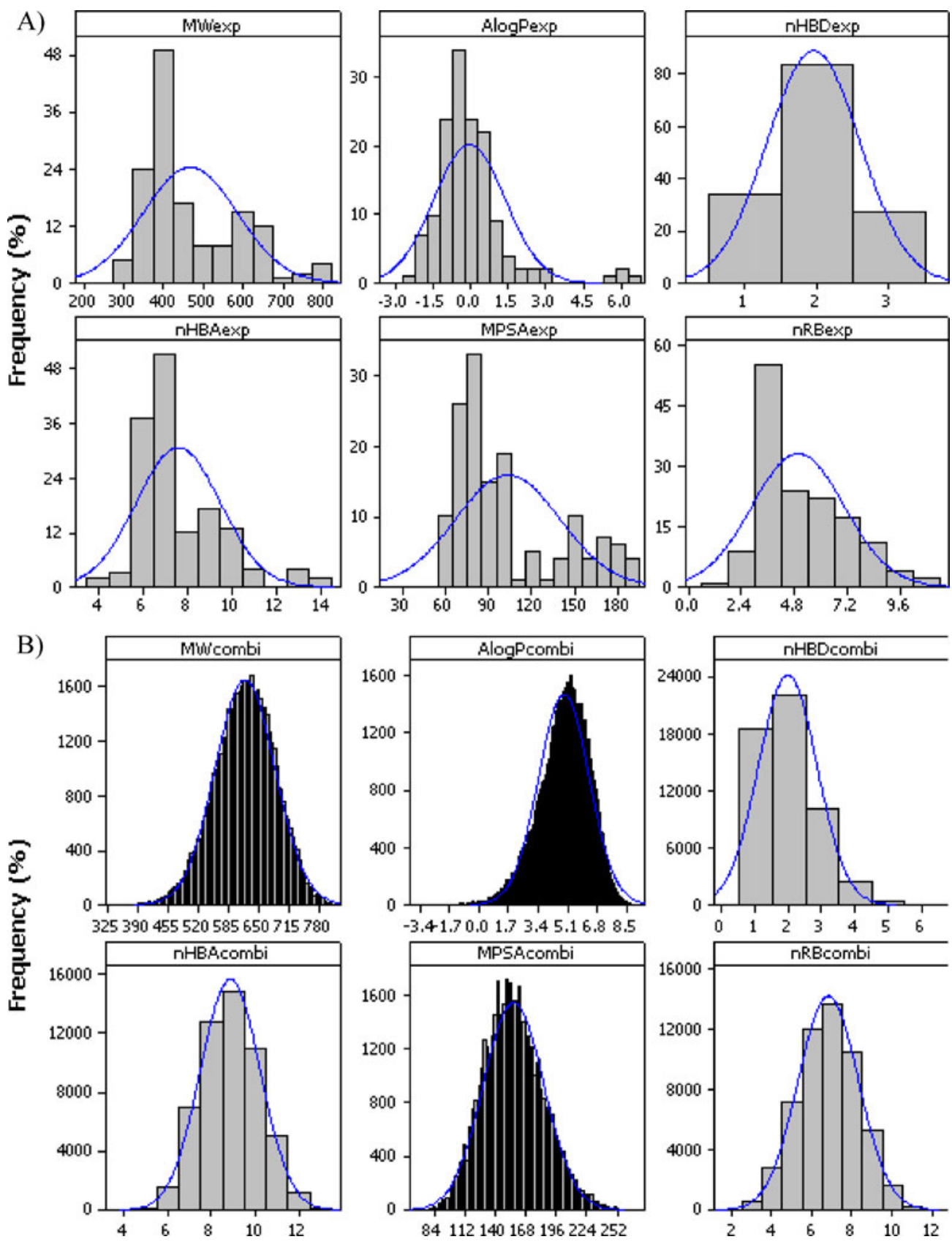

Fig. 4 Histogram-type of analysis for druggability properties assessment (property distribution). (A) the experimental set (Assay2, 145 compounds), (B) the combinatorial set (CombiTot, 53.871 com- pounds). The property distribution is fitted with normal distribution to the histogram of data
(LBP, SBP shared, and $\mathrm{SPB}_{\text {merged }}$ ) were constructed as described previously. Since the automatic pharmacophore generation yielded all possible pharmacophoric features, existing knowledge about the SAR of 6-FQs [16] was employed for pharmacophoric simplification of all models. Such a simplification approach resulted in a total of five pharmacophoric features per model (one aromatic ring, two hydrophobic features, one hydrogen bond donor, and one negative ionizable area). The obtained three-dimensional pharmacophore models (LBP, $\mathrm{SBP}_{\text {shared, }}$, and $\left.\mathrm{SPB}_{\text {merged }}\right)$, served as highly effective in silico filtering tools for subsequent VS of the 6-FQ ligands (Fig. 5).

Since the core idea of the three-dimensional pharmacophoric concept is the selection of active compounds among a pool of inactive molecules [18], our generated 
3D pharmacophore models (LBP, $\mathrm{SBP}_{\text {shared, and }}$ $\mathrm{SPB}_{\text {merged }}$ ) were validated for their recognition performances as in silico filters in a VS experiment using a dataset of known 6-FQs with experimentally-measured biological activity values (Assay2, 145 compounds) [21]. Initially, the LBP model identified 58 active out of total 145 compounds, while the two SBP models $\left(\mathrm{SBP}_{\text {shared }}\right.$ and $\mathrm{SPB}_{\text {merged }}$ ) identified 62 and 49 compounds, as active compounds respectively. The experimentally-determined biological activity values for the successfully filtered compounds are in the range between $0.001<\mathrm{MIC}_{\exp }[\mu \mathrm{g} /$ $\mathrm{mL}]<3.500$, of which around $90 \%$ were determined as highly-active compounds with biological activity values in the range between $0.001<\mathrm{MIC}_{\exp }[\mu \mathrm{g} / \mathrm{mL}]<0.900$. The 6FQs obtained by the pharmacophore-based VS procedure (LBP (58), $\mathrm{SBP}_{\text {shared }}$ (62), and $\mathrm{SBP}_{\text {merged }}$ (49)), were visually inspected for molecular fitness within each of the pre-defined pharmacophoric features of the models (one aromatic ring, two hydrophobic features, one hydrogen bond donor, and one negative ionizable area).

In order to assess the visual inspection more precisely, a Boolean-type of signing (T/F (true/false)) was introduced. Each visually-determined match between the 6-FQ's substructural elements and the pharmacophore-model features was marked as true $(\mathrm{T})$. In addition, the biological activity values $\left(\mathrm{MIC}_{\text {exp }}\right)$ of the investigated compounds were implemented as a feature in the evaluation process using the GHA range. Thus, the compounds with biological activity values in the GHA range were marked as true $(\mathrm{T})$, while the rest of the compounds were marked as false (F). Therefore, only the compounds with (T) outcome for all the pre-defined pharmacophoric-model features as well as lying within the GHA range (electronic supplementary material, online resource 5, experimental set), can be marked as highly favorable 6-FQs (LBP (20 highly active 6-FQs out of 58), $\mathrm{SBP}_{\text {shared }}$ (19 highly active 6-FQs out of 62), and $\mathrm{SPB}_{\text {merged }}$ (8 highly active 6-FQs out of 49 ). Online resource 5, shows that selected compounds from the experimental set (Structure24, Structure39, Structure75, and Structure110), which are all potent compounds, were identified as active compounds by all three pharmacophore models.

The structural analysis of the selected compounds (online resource 5, see "experimental set" sheet), shows that the cyclopropyl group is the most frequently used functional group at position 1 of the main 6-FQ scaffold, whereas position 7 can be successfully substituted by a range of substructural fragments, mainly heterocyclic systems of which 5- and 6-membered N-hetero systems (aminopyrolidines, piperazines), are the most optimal for anti-mycobacterial activity. These results suggest the SAR rules for optimal anti-mycobacterial activity of the 6-FQs [16] and that the three-dimensional pharmacophore concept can be successfully employed as a highly-effective in silico filtering tool [19].

Following the validation on the experimental set [21], the same 3D pharmacophore models were used for assessing the combinatorially-generated subset (CombiLib, 427 novel compounds). The LBP model initially identified 95 active out of a total of 427 compounds as hits, whereas the two structure-based pharmacophore models $\left(\mathrm{SBP}_{\text {shared }}\right.$ and $\left.\mathrm{SBP}_{\text {merged }}\right)$ identified 95 and 77 compounds, respectively. Since the combinatorial subset was built defining a GHA range, all of the 6-FQ analogs in such a library are hypothetically active against $M$. tuberculosis, regarding the $\mathrm{MIC}_{\text {pred-combi }}$ values $\left(0.0021<\mathrm{MIC}_{\text {pred-combi }}[\mu \mathrm{g} / \mathrm{mL}]<0.1000\right)$. Therefore, a visual determination of the matches using $(\mathrm{T} / \mathrm{F})$ designation between the structural elements of the novel actively-recognized 6-FQs hits (LBP (95), $\mathrm{SBP}_{\text {shared }}$ (95), and $\mathrm{SBP}_{\text {merged }}(77)$ ) and pharmacophoric features within the models was implemented to identify the most optimal structural features.

The selected compounds (LBP (32 out of 95), $\mathrm{SBP}_{\text {shared }}$ (26 out of 95), and $\mathrm{SBP}_{\text {merged }}$ (31 out of 77)), belong to three general classess of combinatorially-generated 6-FQ compounds: ciprofloxacin analogs (7-amino $\left(\mathrm{CIP}-N_{i}-M_{j}\right) / 7-$ nonamino derivatives $\left(\mathrm{CIP}^{\prime}-N_{i}-M_{j}\right)$ ), moxifloxacin analogs (7-nonamino derivatives $\left(\mathrm{MOX}^{\prime}-N_{i}-M_{j}\right)$ ) and ofloxacin analogs (7-amino (OFL- $\left.M_{j}\right) / 7$-nonamino derivatives $\left.\left(\mathrm{OFL}^{\prime}-M_{j}\right)\right)$.

The frequency analysis of occurrence of the substructural fragments (throughout the models output,

Table 3 The most important 2D molecular descriptors (pharmacophore fingerprints) for the activity ( $\mathrm{T}$, topological; $\mathrm{C}$, constitutional; $\mathrm{E}$, electrostatic)

\begin{tabular}{|c|c|c|c|c|}
\hline ID & Descriptor & Source & Definition & Class \\
\hline 1. & GATS8v & DRAGON & $\begin{array}{l}\text { Geary autocorrelation- } \\
\text { lag8/weighted by atomic } \\
\text { van der Waals volumes }\end{array}$ & $\mathrm{T}$ \\
\hline 2. & nR09 & DRAGON & $\begin{array}{l}\text { Number of 9-membered } \\
\text { rings }\end{array}$ & $\mathrm{C}$ \\
\hline 3. & JGI2 & DRAGON & $\begin{array}{l}\text { Mean topological charge } \\
\text { index of order } 2\end{array}$ & $\mathrm{E} / \mathrm{T}$ \\
\hline 4. & JGI3 & DRAGON & $\begin{array}{l}\text { Mean topological charge } \\
\text { index of order } 3\end{array}$ & $\mathrm{E} / \mathrm{T}$ \\
\hline 5. & YZS/YZR & CODESSA & $\begin{array}{l}\text { YZ Shadow/YZ } \\
\text { Rectangle }\end{array}$ & $\mathrm{T}$ \\
\hline 6. & $\mathrm{X} 1 \mathrm{~A}$ & DRAGON & $\begin{array}{l}\text { Average connectivity } \\
\text { index chi-1 }\end{array}$ & $\mathrm{T}$ \\
\hline 7. & PW3 & DRAGON & $\begin{array}{l}\text { Path/Walk 3-Randic } \\
\text { shape index }\end{array}$ & $\mathrm{T}$ \\
\hline
\end{tabular}


Fig. 5 The three pharmacophore models obtained after pharmacophoric features reduction (a total of five features per model). (A) ligand-based pharmacophore (LBP) model, (B) structure-based shared pharmacophore $\left(\mathrm{SBP}_{\text {shared }}\right)$ model, $(\mathrm{C})$ structure-based merged pharmacophore $\left(\mathrm{SBP}_{\text {merged }}\right)$ model, (D) The most promising six combinatorial compounds obtained after pharmacophore models VS campaign and aligned into the $\mathrm{SBP}_{\text {shared }}$ pharmacophore model
A)

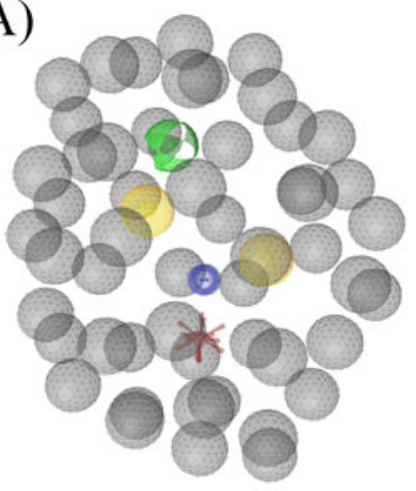

D)

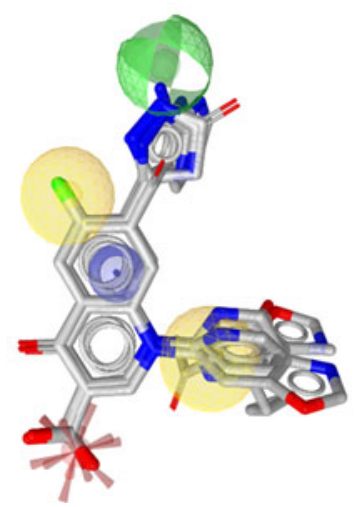

B)

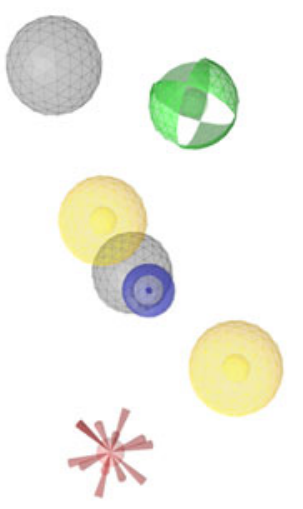

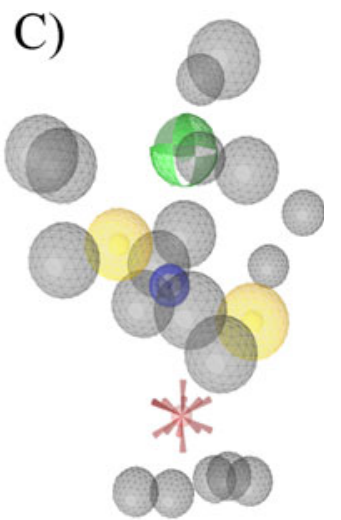

electronic supplementary material, online resource 5, see "combinatorial set" sheet) attached at positions 1 and 7 of the main 6-FQ core - explicitly shows that the most frequently appearing fragment at position 1 (18 times) is the building-block benzo $[d]$ oxazole marked as $N_{i}=028$ in the CIP'-analogs and $N_{i}=016$ in the $\mathrm{MOX}^{\prime}$-analogs, respectively, while the most frequently appearing fragments at position 7 of the main scaffold are the buildingblocks: $1 H$-pyrazolo[3,4-b]pyridine (16 times) marked as $M_{j}=148$ in the CIP'-analogs, $M_{j}=116$ in the MOX'analogs, and $M_{j}=147$ in the $\mathrm{OFL}^{\prime}$-analogs, respectively, and $1 H$-pyrazol-5(4H)-one ( 15 times) marked as $M_{j}=186$ in the CIP'-analogs and $M_{j}=144$ in the MOX'-analogs. In summary, six compounds were selected (CIP'-028-148, CIP'-028-186, MOX'-016-116, MOX'-016-144, OFL'147, and OFL-148) as a result of pharmacophore modeling assessment (Table 4).

Interestingly, these compounds (except for OFL-148, i.e., an ofloxacin analog) belong to the 6-FQ analogs structurally different from ciprofloxacin and moxifloxacin with non-amino substructural fragments at position 7. The predicted biological activity values for these 6-FQ analogs are in the range $0.0035<\mathrm{MIC}_{\text {pred- }}$ combi $[\mu \mathrm{g} / \mathrm{mL}]<0.1000$. The selected combinatoriallygenerated 6-FQ analogs, were subsequently used for assessing the possible interactions with experimentallybound 6-FQ protein structure, as an integrated part of the external combinatorial subset (CombiLib, 427 novel compounds) in the molecular docking study.

\section{Molecular docking study}

The molecular docking study on both, the experimental set (Assay2, 145 compounds) with experimentallydetermined biological activity values and the combinatorial one (CombiLib, 427 compounds), was performed by using the experimentally-determined 6-FQs binding site of the type II topoisomerase protein (3K9F). In a previously published docking study, only GyrA protein subunit without full complex details (DNA and GyrB position), was used to assess the possible binding modes [49]. The co-crystallized 6-FQ inhibitor levofloxacin [38] was used for comparison as well as to analyze the geometric and structural properties of the docked 6-FQ compounds in the protein. The re-docking validation procedure [40] of levofloxacin into its binding site was successful as GOLD was able to reproduce the experimental bound conformation with high accuracy.

The post-docking VS analysis was divided into two levels. At the first level, the geometric properties of both sets of compounds were assessed by visually inspecting each 6-FQ dock position relative to the experimental conformation of levofloxacin [38]. The following geometric properties were compared: the visual 
Table 4 The combinatorial compounds selected according to the frequency analysis of the sub-structural fragments (throughout the pharmacophore models output) attached at position $R_{1}$ and $R_{7}$ of the main 6-FQ scaffold, respectively, together with their corresponding pharmacophore fitness score (PFS). The selected compounds with highest predicted biological activity values $\left(\mathbf{M I C}_{\text {pred-combi }}\right)$, are highlighted in gray

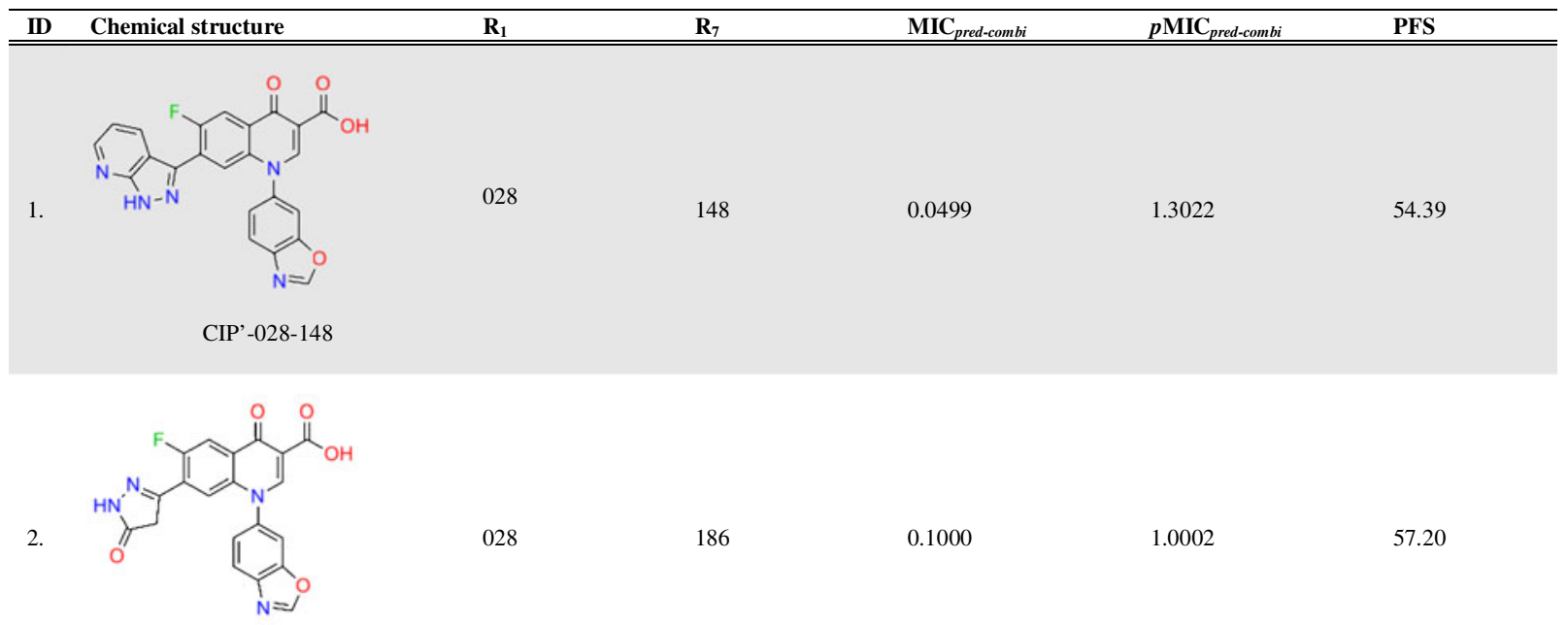

CIP'-028-186

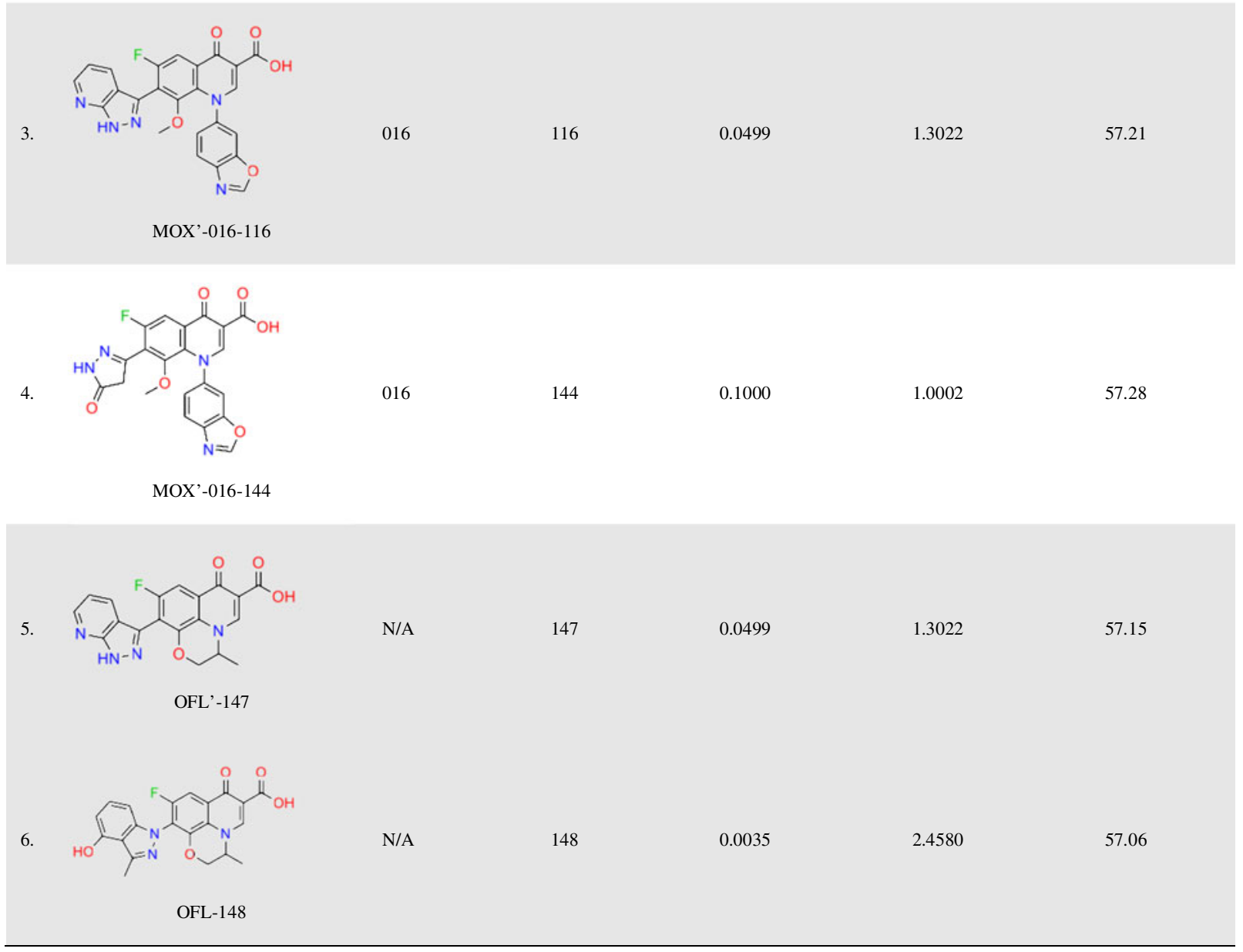

orientation (how the screened 6-FQ compound is oriented relative to the levofloxacin position), visual fitness (how well the screened 6-FQ compound fits the experimental levofloxacin conformation), and the number of the matching pharmacophoric features (how many common pharmacophoric features, generated for each docked 
conformation, are shared by both screened 6-FQ compound and levofloxacin). Similarly to the pharmacophore analysis, a Boolean-type of signing (T/F (true/false)) was employed for assessing the visual orientation and visual fitness parameters, while the matching pharmacophoric features were defined numerically. The screened compounds which share 4-5 pharmacophoric features present in bound levofloxacin, were signed as true (T). Therefore, the result of the first level assessment can be defined as a sum of the obtained Boolean answers for all three investigated properties (electronic supplementary materials, online resource 6).

First level post-docking analysis of the experimental set of 6-FQ analogs docked into the $3 \mathrm{~K} 9 \mathrm{~F}$ binding pocket (online resource 6, see "Exp-3K9F" sheet) resulted in 102 (T) marked compounds out of 145 , of which 38 compounds have measured biological activity values within $0.001<\mathrm{MIC}_{\text {exp }}$ $[\mu \mathrm{g} / \mathrm{mL}]<0.1$. Moreover, as shown in Fig. $6 \mathrm{~A}$, a favorable positioning of these 6-FQ compounds into the $3 \mathrm{~K} 9 \mathrm{~F}$ binding pocket could be observed. The structural analysis of these 38 compounds, once again showed that the cyclopropyl group is the most frequently appearing substituent at position 1 of the main 6-FQ scaffold (33 times), while the piperazinyl group attached at position 7 appeared 24 times. These results are again in accordance with the previous experimental SAR findings (online resource 6, "experimental set" sheet) [16].

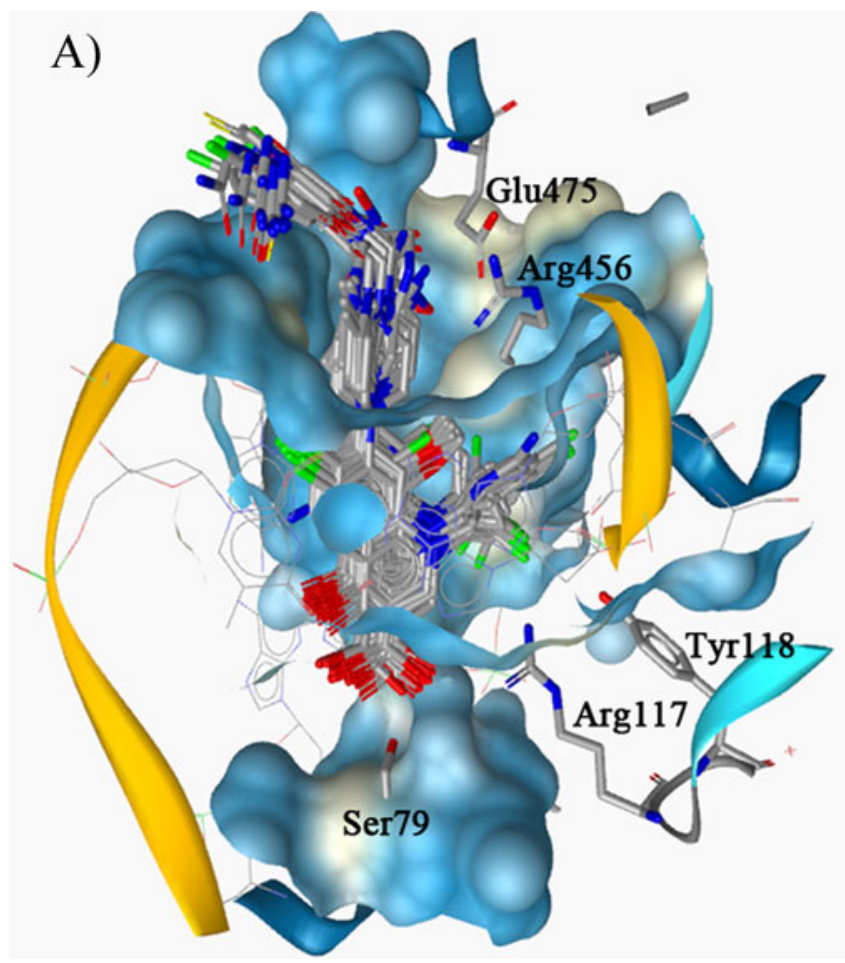

Fig. 6 Results of docking into the 3K9F protein binding pocket. (A) 38 compounds from the experimental set with acceptable geometric properties. (B) 166 compounds from the combinatorial set with
The first level post-docking analysis of the 6-FQ analogs in the combinatorial set (CombiLib, 427 compounds) docked into the $3 \mathrm{~K} 9 \mathrm{~F}$ binding pocket (electronic supplementary materials, online resource 6, see "Combi-3K9F" sheet) resulted in $166(\mathrm{~T})$ marked compounds out of 427.

Interestingly, comparing with the previous assessment using pharmacophore models filters where none of the MOX $-N_{i}-M_{j}$ analogs was recognized as active for further investigation, the post-docking Boolean procedure performed here identified only one moxifloxacin analog (MOX-028-064) as geometrically suitable 6-FQ compound in $3 \mathrm{~K} 9 \mathrm{~F}$ binding pocket. The $6-\mathrm{FQ}$ combinatorial hit analogs belonging to the other five structural classes (CIP$N_{i}-M_{j}, \mathrm{CIP}^{\prime}-N_{i}-M_{j}$, MOX'- $N_{i}-M_{j}$, OFL- $M_{j}$, OFL'- $M_{j}$ ) were successfully identified. According to the combinatorial 6-FQ (T) outcome obtained, 166 compounds from the combinatorial set have geometric properties, comparable to the co-crystallized levofloxacin (Fig. 6B).

The frequency analysis of occurrence of the substructural fragments attached at positions 1 and 7 of these 166 compounds, indicated that the most frequently appearing fragments at position 1 are the building-blocks: benzo $[d]$ oxazole (46 times) marked as $N_{i}=028$ in the CIP'-analogs and in the CIP-analogs, and $N_{i}=016$ in the MOX'-analogs, respectively, and 1-(pyridin-3-yl)ethanone (11 times) marked as $N_{i}=102$. The most frequently appearing frag-

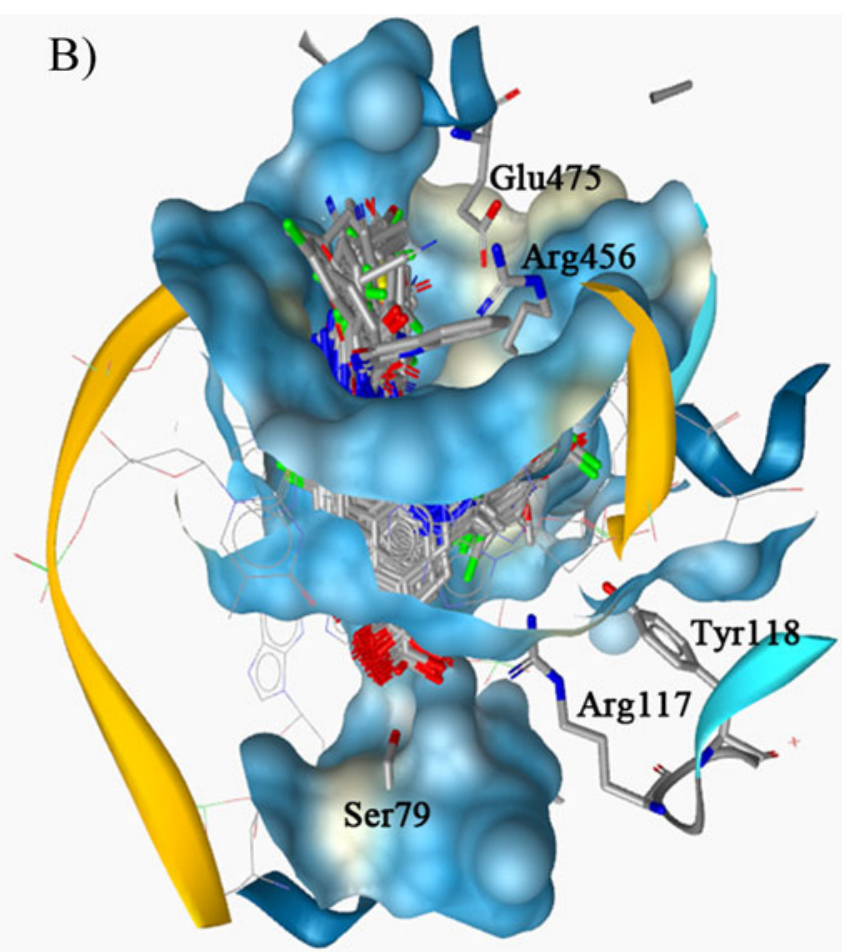

acceptable geometric properties. Crucial amino acid residues from the 3K9F 6-FQs binding site are displayed [35] 
ments at position 7 of the main scaffold are the buildingblocks: $4 H$-furo[3,2-c]pyran-4-one (8 times) marked as $M_{j}=006,1 H$-pyrazolo[3,4-b]pyridine (7 times ) marked as $M_{j}=148$, and $1 H$-pyrazole ( 7 times) marked as $M_{j}=176$.

Except compound OFL-148, all selected 6-FQs from the performed pharmacophore analysis $\left(\mathrm{CIP}^{\prime}-028-148, \mathrm{CIP}^{\prime}\right.$ 028-186, MOX'-016-116, MOX'-016-144, OFL'-147) were successfully identified as compounds with favorable threedimensional binding geometry as well (online resource 6, see "Combi-3K9F" sheet). Therefore, all of the selected 6FQ analogs from the experimental set (3K9F (102)) and the combinatorial one (3K9F (166)), respectively, were subsequently used for further investigation at the second level post-docking analysis.

At the second level of the post-docking analysis, the intermolecular interaction properties of the selected geometrically suitable 6-FQs from both experimental and combinatorial sets were investigated (electronic supplementary materials, online resource 8 ). The crystal structure of the S. pneumoniae topoisomerase IV protein in complex with the co-crystallized ligand levofloxacin (3K9F) was used as a starting point for measuring the interaction distances between the ligand's functional groups and the surrounding amino acid residues [38]. A set of five key interactions between the levofloxacin and the protein were used as standard measures: $\left(\mathrm{R}_{3}-\mathrm{COO}^{-}\right)$ $(\mathrm{HO}-\mathrm{Ser} 79) \quad\left[d_{3 \text { K } 9 \text {-ref }-1}=3.18 \AA\right],\left(\mathrm{R}_{3}-\mathrm{COO}^{-}\right)-(\mathrm{HO}-$ Ser79) $\left[d_{3 K 9 F-r e f-2}=3.35 \AA\right],\left(\mathrm{R}_{3}-\mathrm{COO}^{-}\right)-(\mathrm{HO}-\mathrm{Arg} 117)$ $\left[\begin{array}{ll}d_{3 K 9 F-r e f-3}=3.01 & \AA\end{array}\right], \quad\left(\mathrm{R}_{7}=\mathrm{N}-\mathrm{CH}_{3}\right)-\left(\mathrm{H}_{2} \mathrm{~N}^{+}=\mathrm{Arg} 456\right)$ $\left[\begin{array}{ll}d_{3 K 9 F-r e f-4}=3.30 \AA & \AA\end{array}\right.$, and $\left(\mathrm{R}_{7}=\mathrm{N}-\mathrm{CH}_{3}\right)-\left({ }^{-O O C}-\mathrm{Glu} 475\right)$ $\left[d_{3 K 9 F-r e f-5}=4.38 \AA\right]$. Since the biological systems are not static and are characterized with dynamic features, a distance tolerance of \pm 1 Angstrom unit around each of the standard interatomic distance values was employed: $\left(\mathrm{R}_{3}-\mathrm{COO}^{-}\right)-(\mathrm{HO}-\mathrm{Ser} 79) \quad\left[d_{3 K 9 F-1}=2.18-4.18 \AA\right],\left(\mathrm{R}_{3}-\right.$ $\left.\mathrm{COO}^{-}\right)-(\mathrm{HO}-\mathrm{Ser} 79)\left[d_{3 K_{9-2}-2}=2.35-4.35 \AA\right],\left(\mathrm{R}_{3}-\mathrm{COO}^{-}\right)-$ $(\mathrm{HO}-\mathrm{Arg} 117) \quad\left[d_{3 K 9 F-3}=2.01-4.01 \AA\right], \quad\left(\mathrm{R}_{7}=\mathrm{N}-\mathrm{CH}_{3}\right)-$ $\left(\mathrm{H}_{2} \mathrm{~N}^{+}=\operatorname{Arg} 456\right)\left[d_{3 K 9 F-4}=2.30-4.30 \AA\right]$, and $\left(\mathrm{R}_{7}=\mathrm{N}-\right.$ $\left.\mathrm{CH}_{3}\right)$-('OOC-Glu475) $\left[d_{3 K 9 F-5}=3.38-5.38 \AA\right]$.

The second level post-docking analysis in 3K9F (online resource 7, see "Exp-3K9F" sheet) selected 45 out of 102 compounds from the experimental set as optimal 6-FQ compounds (regarding the interatomic distances). The biological activity values for these compounds are in the range between $0.001<\mathrm{MIC}_{\exp }[\mu \mathrm{g} / \mathrm{mL}]<6.700$ of which approximately $38 \%$ have good measured biological activity $\left(0.001<\mathrm{MIC}_{\exp }[\mu \mathrm{g} / \mathrm{mL}]<0.100\right)$. The structural analysis once again showed that the most frequently occuring substituent at position 1 of the main 6-FQ scaffold is the cyclopropyl group (15 times), whereas the most frequent substituent attached at position 7 of the hit molecules is the piperazinyl group (12 times), whose results are in accordance with the established 6-FQs SAR rules (Fig. 1) [16].
The second level post-docking analysis of the combinatorial compounds resulted in selection of a total of 11 out of 166 compounds (online resource 7, see "Combi-3K9F" sheet). These compounds mainly belong to the class of 7 nonamino substituted ciprofloxacin analogs $\mathrm{CIP}^{\prime}-N_{i}-M_{j}$ (nine compounds), one 7-amino substituted ciprofloxacin analog $\left(\mathrm{CIP}-N_{i}-M_{j}\right)$, and one 7-amino substituted ofloxacin analog $\left(\mathrm{OFL}-M_{j}\right)$. No moxifloxacin analogs $\left(\mathrm{MOX}-N_{i}-M_{j}\right.$ and $\left.\mathrm{MOX}^{\prime}-N_{i}-M_{j}\right)$ and 7-nonamino substituted ofloxacin analogs $\left(\mathrm{OFL}^{\prime}-M_{j}\right)$ were found that could be fitted in this set of defined interatomic distance boundaries [38].

The frequency analysis of occurrence of the substituents attached at position 1 and 7 of the main scaffold, once again pinpointed the fragment marked as $N_{i}=028$ as the most frequently appearing substituent at position 1 (5 times), while position 7 can be successfully substituted with a range of different groups. Incorporation of these fragments thus forms novel 6-FQ compounds that could also serve as novel target compounds in the synthetically-driven lead optimization. The list of compounds that complements the previous selection in Table 4 is presented in Table 5.

\section{Conclusions}

In the present study a variety of chemometric and molecular modeling approaches were integrated into a powerful complex scheme capable of constructing as well as evaluating a virtual combinatorial library of 6-fluoroquinolone analogs (6-FQs). This 6-FQs library was designed by employing the synthetically-driven ligand generation rules feasible of yielding ligands which can be readily synthesized and have a predicted inhibitory activity toward GyrA.

The results can be summarized as follows: (1) a large number of virtual 6-fluoroquinolone analogs $(53.871 \mathrm{com}$ pounds) were generated by a combinatorial generation of all substituted amine and non-amine compounds at positions 1 and 7, respectively. The selection of a drug-like set of 427 compounds from the library within the GHA range was performed by using drug-likeness filters (a combined Lipinski-Veber filtering tool based on the Lipinski's and Veber's rule-sets for drug-likeness) and our previously developed and validated neural-networks (NN) chemometric model (built on a dataset of structurally-similar 6FQ compounds with experimentally-determined biological activity values by employing a combined QSAR modeling strategy, i.e., linear modeling as well as non-linear modeling using Kohonen and counter-propagation artificial neural networks) [21]. (2) Experimental data on the structurallysimilar topoisomerase-IV enzyme in complex with levofloxacin was used to construct structure-based as well as ligand-based pharmacophore models to evaluate the most promising 6-FQs obtained by chemometric methods. The 
Table 5 The combinatorial compounds extracted as most suitable after the second level VS analysis into the $3 \mathrm{~K} 9 \mathrm{~F}$ binding pocket, together with their GoldScore Fitness (GSF) scoring function and the corresponding inter-atomic distances (in Ångstrom units) between the
6-FQ sub-structural fragments and the crucial amino acid residues important for the biological activity. The compounds with highest predicted biological activity values $\left(\mathbf{M I C}_{\text {pred-combi }}\right)$, are highlighted in gray

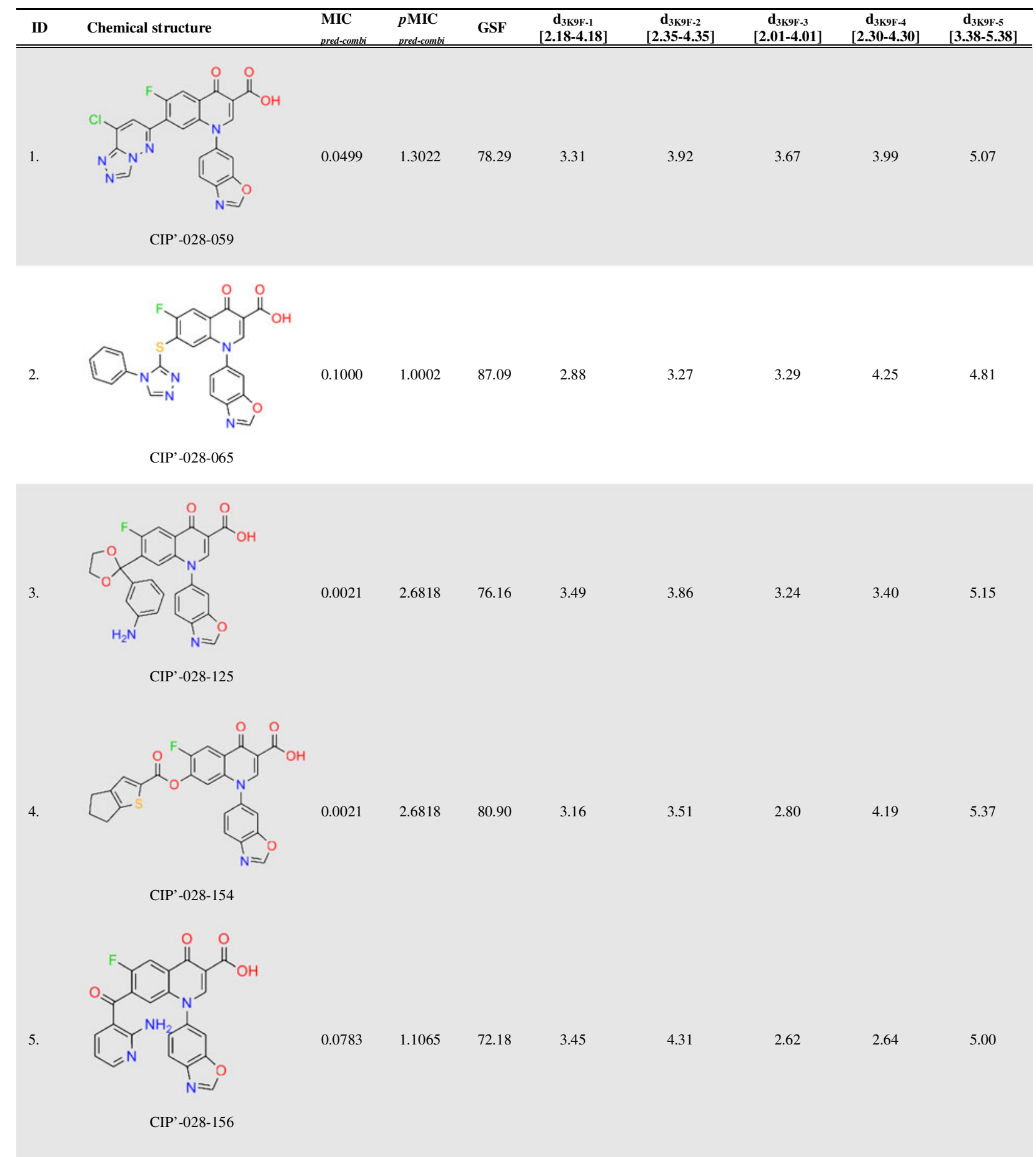

models were also validated using available experimental 6-FQs data from the literature. (3) Finally, these compounds were docked into the topo-IV binding pocket and the interactions of 6-FQ analogs from virtual library as well as experimental database with the surrounding amino acid residues were compared and analyzed.

The outcome of this study shows that promising compounds for further investigation originate from all three 
Table 5 (continued)

6.<smiles>O=C(O)c1cn(-c2ccc(O)c(CO)c2)c2cc(-c3cc4occc4c(=O)o3)c(F)cc2c1=O</smiles>

CIP'-037-006 $\begin{array}{llllllll}0.0130 & 1.8851 & 72.26 & 3.17 & 3.51 & 3.31 & 3.92 & 3.43\end{array}$

$\begin{array}{llllllll}0.1000 & 1.0002 & 71.96 & 2.96 & 3.84 & 3.92 & 4.08 & 3.96\end{array}$

7.<smiles>Nc1c(-c2cc3c(cc2F)c(=O)c(C(=O)O)cn3-c2nccc(C3CC3)n2)ccc2cccnc12</smiles>

CIP'-087-073

8.<smiles>COc1cc(-n2cc(C(=O)O)c(=O)c3cc(F)c(C4=NNC(=O)C4)cc32)ccc1C</smiles>

71.79

3.09

3.51

3.60

3.58

5.33

CIP'-095-186

9.<smiles>CC(=O)c1cccnc1-n1cc(C(=O)O)c(=O)c2cc(F)c(-c3cnn4c(C5CC5)ccnc34)cc21</smiles>
0.1000 $1.0002 \quad 83.74 \quad 3.94$ .94

4.33

CIP'-102-113

10.<smiles>CC(=O)c1cccnc1-n1cc(C(=O)O)c(=O)c2cc(F)c(N3CC(C)=NN3C(=O)O)cc21</smiles>

CIP-103-102

11.

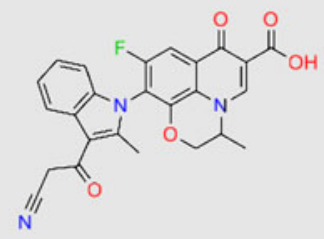

OFL-127 $\begin{array}{llll}0.0681 & 1.1666 \quad 74.36 & 3.7\end{array}$

4.17

2.83

4.29

5.29

1.88

72.53

3.34

3.65

3.00

3.01

3.73 chemical classes (ciprofloxacin, moxifloxacin, and ofloxacin analogs), whereas the ciprofloxacin chemical class yielded the highest number of hits (CIP'-028-059, CIP'028-125, CIP'-028-148, CIP'-028-154, CIP'-028-156, CIP'- 
037-006, CIP'-095-186, and CIP-103-102). Furthermore, the detailed analysis of occurrence of the substructural fragments present at positions $\mathrm{R}_{1}$ and $\mathrm{R}_{7}$ of the 6-FQ hit molecules revealed several novel attractive fragments, such as for $\mathrm{R}_{1}$ : $\left[\left(N_{i}=028\right.\right.$, benzo[ $\left.d\right]$ oxazole), $\left(N_{i}=037,2\right.$-(hydroxymethyl)phenol), ( $N_{i}=095,1$-methoxy-2-methylbenzene), and $\left(N_{i}=103,1\right.$-(pyridin-3-yl)ethanone) $]$ and for $\mathrm{R}_{7}$ : $\left[\left(M_{j}=\right.\right.$ 006, $4 H$-furo[3,2-c]pyran-4-one), $\left(M_{j}=059,8\right.$-chloro-[1, 2, 4] triazolo[4,3- $b]$ pyridazine), $\left(M_{j}=102\right.$, 3-methyl- $1 H$-pyrazol5(4H)-one), $\left(M_{j}=125\right.$, 3-(2-methyl-1,3-dioxolan-2-yl)aniline), $\left(M_{j}=148,1 H\right.$-pyrazolo[3,4- $\left.b\right]$ pyridine $),\left(M_{j}=154\right.$, methyl 5,6-dihydro-4H-cyclopenta[b]thiophene-2-carboxylate), $\left(M_{j}=156,1-(2\right.$-aminopyridin-3-yl)ethanone), and $\left(M_{j}=186,1 H\right.$-pyrazol-5(4H)-one], respectively, that satisfied the screening conditions at all levels. In conclusion, we hope that the results of our mixed chemometricmolecular modeling study will assist in providing new SAR guidelines to the lead optimization stage of the 6FQ's drug design and thus enable the design of the novel much-needed - antibacterial agents.

Acknowledgments Authors thank Agency of Research of R. Slovenia (ARRS) for the financial support through the Grants P10017 and 1000-07-310016. We are sincerely grateful to Dr. Marjana Novič for valuable insights, discussion and her continuing support of this research.

\section{References}

1. Munro SA, Lewin SA, Smith HJ, Engel ME, Feetheim A, Volmink J (2007) Patient adherence to tuberculosis treatment: a systematic review of qualitative research. PloS Med 4:1230-1245

2. Du Toit LC, Pillay V, Danckwerts MP (2006) Tuberculosis chemotherapy: current drug delivery spproaches. Respir Res 7:118-136

3. Bhanu NV, van Soolingen D, van Embden JDA, Seth P (2004) Two Mycobacterium fortuitum strains isolated from pulmonary tuberculosis patients in Delhi IS6110 harbour homologue. Diagn Micro Infec Dis 48:107-110

4. Dussurget O, Rodriguez M, Smith I (1998) Protective role of Mycobacterium smegmatis IdeR against reactive oxygen species and isoniazid toxicity. Tubercle Lung Dis 79:99-106

5. Field SK, Fisher D, Cowie RL (2004) Mycobacterium avium complex pulmonary disease in patients without HIV infection. Chest 126:566-581

6. Zhang Y, Martens KP, Denkin S (2006) New drug candidates and therapeutic targets for tuberculosis therapy. Drug Discovery Today $11: 21-27$

7. Wigley DB (1995) Structure and mechanism of DNA gyrase. In: Eckstein F, Lilley DMJ (eds) Nucleic Acids Molecular Biol. Springer, Berlin, pp 165-176

8. Barnard FM, Maxwell A (2001) Interaction between DNA gyrase and quinolones: effects of alanine mutations at GyrA subunit residues $\mathrm{Ser}^{83}$ and Asp ${ }^{87}$. Antimicrob Agents Chemother 45:1994-2000

9. Peng H, Marians KJ (1993) Escherichia coli topoisomerase IV. Purification, characterization, subunit structure, and subunit interactions. J Biol Chem 268:24481-24490

10. Reece RJ, Maxwell A (1991) DNA gyrase: structure and function. Crit Rev Biochem Mol 26:335-375
11. Levine C, Hiasa H, Marians KJ (1998) DNA gyrase and topoisomerase IV: biochemical activities, physiological roles during the chromosome replication, and drug sensitivities. Biochim Biophys Acta 1400:29-43

12. Ostrov DA, Hernández Prada JA, Corsino PE, Finton KA, Le N, Rowe TC (2007) Discovery of novel DNA gyrase inhibitors by high-throughput virtual screening. Antimicrob Agents Chemother 51:3688-3698

13. Zhang Y (2005) The magic bullets and tuberculosis drug targets. Annu Rev Pharmacol Toxicol 45:529-564

14. Vashist J, Vishvanath KR, Kapil A, Yennamalli Y, Subbarao N, Rajeswari MR (2009) Interaction of nalidixic acid and ciprofloxacin with wild type and mutated quinolone-resistance-determining region of DNA gyrase A. Indian J Biochem Biophys 46:147-153

15. Hooper DC (1999) Mode of action of fluoroquinolones. Drugs 58 (suppl 2):6-10

16. Peterson LR (2001) Quinolone molecular structure-activity relationships: what we have learned about improving antimicrobial activity. Clin Infect Dis 33(Suppl 3):S180-S186

17. Ebalunode JO, Zheng W, Tropsha A (2011) Application of QSAR and shape pharmacophore modeling approaches for targeted chemical library design. Methods Mol Biol 685:111-133

18. Wolber G, Langer T (2005) LigandScout: 3-D pharmacophores derived from protein-bound ligands and their use as virtual screening filters. J Chem Inf Comput Sci 45:160-169

19. Sippl W (2008) Pharmacophore identification and pseudo-receptor modeling. In: Wermuth CG (ed) The Practice of Medicinal Chemistry, 3rd edn. Academic Press/Elsevier, Amsterdam, pp 572-586

20. Perdih A, Kovač A, Wolber G, Blanot D, Gobec S, Solmajer T (2009) Discovery of novel benzene 1,3-dicarboxylic acid inhibitors of bacterial MurD and MurE ligases by structure-based virtual screening approach. Bioorg Med Chem Lett 19:26682673

21. Minovski N, Vračko M, Šolmajer T (2010) Quantitative structureactivity relationship study of antitubercular fluoroquinolones. Mol Div 15:417-426

22. http://www.cambridgesoft.com/software/ChemBioOffice

23. Schwalbe T, Kadzimirisz D, Jas G (2000) Synthesis of a library of ciprofloxacin analogues by means of sequential organic synthesis in microreactors. QSAR Comb Sci 24:758-768

24. Martel AM, Leeson PA, Castañer J (1997) BAY-12-8039: fluoroquinolone antibacterial. Drugs Fut 22:109-113

25. Serradell MN, Blancafort P, Castañer J (1983) DL-8280. Drugs Fut 8:395

26. Key Organics, Bionet Fragment Library "Rule of 3", http://www. keyorganics.ltd.uk

27. Congreve M, Carr R, Murray CW, Jhoti H (2003) A rule of three for fragment-based lead discovery? Drug Discovery Today 8:876877

28. Minovski N, Šolmajer T (2010) Chemometrical exploration of combinatorially generated drug-like space of 6-fluoroquinolone analogs: a QSAR study. Acta Chim Slov 57:529-591

29. Wieland T (1997) Combinatorics of combinatorial chemistry. J Math Chem 21:141-157

30. Bohacek RS, mcMartin C, Guida WC (1996) The art and practice of structure-based drug design. Med Res Rev 16:3-50

31. Lipinski CA, Lombardo F, Dominy BW, Feeney PJ (1997) Experimental and computational approaches to estimate solubility and permeability in drug discovery and development settings. Adv Drug Deliv Rev 23:3-25

32. Veber DF, Johnson SR, Cheng H-Y, Smith BR, Ward KW, Kopple KD (2002) Molecular properties that influence the oral bioavailability of drug candidates. J Med Chem 45:26152623

33. Katritzky AR, Lobanov VS, Karelson M (1995) CODESSA. Reference manual, University of Florida, Gainsville 
34. Tretter EM, Schoeffler AJ, Weisfield SR, Berger JM (2010) Protein Struct Funct Bioinf 78:492-495

35. Fu G, Wu J, Liu W, Zhu D, Hu Y, Deng J, En Zhang X, Bi L, Cheng Wang D (2009) Crystal structure of DNA gyrase B' domain sheds lights on the mechanism for T-segment navigation. Nucleic Acids Res 37:5908-5916

36. Laponogov I, Sohi MK, Veselkov DA, Pan XS, Sawhney R, Thompson AW, McAuley KE, Fisher LM, Sanderson MR (2009) Structural insight into the quinolone-DNA cleavage complex of type IIA topoisomerases. Nat Struct Mol Biol 16:667-669

37. Kitamura A, Hoshino K, Kimura Y, Hayakawa I, Sato K (1995) Contribution of the C-8 substituent of DU-6859a, a new potent fluoroquinolone, to its activity against DNA gyrase mutants of Pseudomonas aeruginosa. Antimicrob Agents Chemoter 39:14671471

38. Laponogov I, Pan XS, Veselkov DA, McAuley KE, Fisher LM, Sanderson MR (2010) Structural basis of gate-DNA breakage and resealing by type II topoisomerases. PloS One 5:e11338(1-8)

39. Jones G, Willet P, Glen RC, Leach AR, Taylor R (1997) Development and validation of a genetic algorithm for flexible docking. J Mol Biol 267:727-748

40. Kirchmair J, Markt P, Distinto S, Wolber G, Langer T (2008) Evaluation of the performance of $3 \mathrm{D}$ virtual screening protocols: RMSD comparisons, enrichment assessments, and decoy selection-What can we learn from earlier mistakes? J Comput Aided Mol Des 22:213-228
41. Huc I, Lehn J-M (1997) Virtual combinatorial libraries: Dynamic generation of molecular and supramolecular diversity by selfassembly. Proc Natl Acad Sci 94:2106-2110

42. Oprea TI, Gottfries J, Sherbukhin V, Svensson P, Kühler TC (2000) Chemical information management in drug discovery: Optimizing the computational and combinatorial chemistry interfaces. J Mol Graph Model 18:512-524

43. Langer T, Wolber G (2004) Virtual combinatorial chemistry and in silico screening: Efficient tools for lead structure discovery? Pur Appl Chem 76:991-996

44. Seneci P, Miertus S (2000) Combinatorial chemistry and highthroughput screening in drug discovery: different strategies and formats. Mol Diversity 5:75-89

45. Oprea TI (2002) Chemical space navigation in lead discovery. Curr Opin Chem Biol 6:384-389

46. Oprea TI (2000) Property distribution of drug-related chemical databases. J Comput Aided Mol Des 14:251-264

47. Allu TK, Oprea TI (2005) Rapid evaluation of synthetic and molecular complexity for in silico chemistry. J Chem Inf Model 45:1237-1243

48. Hann MM, Leach AR, Harper G (2001) Molecular complexity and its impact on the probability of finding leads for drug discovery. J Chem Inf Comput Sci 41:856-864

49. Madurga S, Sánchez-Céspedes J, Belda I, Vila J, Giralt E (2008) Mechanism of binding of fluoroquinolones to the quinolone resistance determining region of DNA gyrase: towards an understanding of the molecular basis of quinolone resistance. Chem Bio Chem 9:2081-2086 